Article

\title{
AN-Aided Secure Beamforming in Power-Splitting-Enabled SWIPT MIMO Heterogeneous Wireless Sensor Networks
}

\author{
Weili Ge ${ }^{1 \oplus}$, Zhengyu Zhu ${ }^{1, *} \mathbb{1}$, Wanming Hao ${ }^{1,2}$, Yi Wang ${ }^{3,4}$, Zhongyong Wang ${ }^{1, *}$, \\ Qiong $\mathrm{Wu}^{5,6,7}$ and Zheng $\mathrm{Chu}$ 2,8 \\ 1 School of Information Engineering, Zhengzhou University, Zhengzhou 450000, China; \\ iewlge@zzu.edu.cn (W.G.); w.hao@surrey.ac.uk (W.H.) \\ 2 5G Innovation Center (5GIC), Institute for Communication Systems (ICS), University of Surrey, \\ Guildford GU2 7XH, UK; zheng.chu@surrey.ac.uk \\ 3 School of Electronics and Communication Engineering, Zhengzhou University of Aeronautics, \\ Zhengzhou 450046, China; yiwang@zua.edu.cn \\ 4 National Digital Switching System Engineering and Technological Research Center, \\ Zhengzhou 450002, China \\ 5 Jiangsu Provincial Engineering Laboratory for Pattern Recognition and Computational Intelligence, \\ Jiangnan University, Wuxi 214122, China; qiongwu@jiangnan.edu.cn \\ 6 National Mobile Communications Research Laboratory, Southeast University, Nanjing 210096, China \\ 7 Department of Electronic Engineering, Tsinghua University, Beijing 100084, China \\ 8 The Shaanxi Key Laboratory of Information Communication Network and Security, Xi'an University of \\ Posts \& Telecommunications, Xi'an 710121, Shaanxi, China \\ * Correspondence: iezyzhu@zzu.edu.cn (Z.Z.); iezywang@zzu.edu.cn (Z.W.)
}

Received: 4 March 2019; Accepted: 17 April 2019; Published: 25 April 2019

\begin{abstract}
In this paper, we investigate the physical layer security in a two-tier heterogeneous wireless sensor network (HWSN) depending on simultaneous wireless information and power transfer (SWIPT) approach for multiuser multiple-input multiple-output wiretap channels with artificial noise (AN) transmission, where a more general system framework of HWSN only includes a macrocell and a femtocell. For the sake of implementing security enhancement and green communications, the joint optimization problem of the secure beamforming vector at the macrocell and femtocell, the AN vector, and the power splitting ratio is modeled to maximize the minimal secrecy capacity of the wiretapped macrocell sensor nodes (M-SNs) while considering the fairness among multiple M-SNs. To reduce the performance loss of the rank relaxation from the SDR technique while solving the non-convex max-min program, we apply successive convex approximation (SCA) technique, first-order Taylor series expansion and sequential parametric convex approximation (SPCA) approach to transform the max-min program to a second order cone programming (SOCP) problem to iterate to a near-optimal solution. In addition, we propose a novel SCA-SPCA-based iterative algorithm while its convergence property is proved. The simulation shows that our SCA-SPCA-based method outperforms the conventional methods.
\end{abstract}

Keywords: heterogeneous wireless sensor networks; SWIPT; physical-layer secrecy; secure beamforming; successive convex approximation

\section{Introduction}

Recently, Internet-of-Things (IoT) has emerged to be considered as one of the key technological concepts as a basic precondition implementing Industry 4.0 [1]. The recent developments in the IoT 
application, wireless sensor networks (WSNs), have played a significant role in connecting tens of thousands of wireless devices over the Internet in many applications, especially healthcare services, electronic instrumentation, transportation, and environmental assessment [2]. A WSN generally contains many sensor nodes (SNs) that is capable of perceiving their external environment, which can perform communicate and computations [3]. Unfortunately, because SN are battery-powered, the working lifetime of the entire WSNs is limited by energy consumption due to the energy restriction at SN [4]. In order to overcome these limitation, there are many investigations on enabling technologies for energy sustainablility in WSNs [5].

The emergence of mobile devices has resulted in a continuously growing requirement for wireless applications, such as an ultra-wide radio coverage, an ultra-high data service, and an ultra-large number of smart devices. Recently, a promising solution to cater for this requirement has been the fifth generation (5G) wireless technology [6,7]. Therefore, the deployment of low-cost femtocell base stations (FBSs) underlaid in the macrocell can provide ubiquitous coverage and extremely high data rate [8]. In this regard, to accommodate the explosive cell density and growing data traffic, heterogeneous networks (HetNets) have emerged as one of the most promising approaches in the $5 \mathrm{G}$ wireless systems by increasing the off-load portion of their traffic $[9,10]$.

In recent years, by integrating the WPT with traditional wireless information transmission (WIT), simultaneous wireless information and power transfer (SWIPT) have been recognised as among the main candidate technologies for green communications [11-15], which has been shown to be in a position to improve the WPT performance in HetNets [16-18]. The idea of using SWIPT to implement wireless recharging of the batteries in HetNets was first studied in [16], where two integer linear programming methods were proposed to help wireless nodes to scavenge ambient energy. Tang et al. [17] provided a essential study of the QoS-constrained energy efficiency optimization problem for coordinated multi-point (CoMP)-SWIPT HetNets, a decoupled approach was proposed in which the power allocation procedure and beamforming design were separated. Considering multiple Power splitting (PS)-based heterogeneous users in [18], Jiang et al. investigated the optimal transmit power minimization design problem of SWIPT systems to achieve green communication design under non-linear energy harvesting model, where the semidefinite relaxation (SDR) are employed to solve and the globe optimal solution was theoretically guaranteed.

On the other hand, depending on the presence of the broadcasting nature of wireless channel and the multi-tier dynamic topology in SWIPT HetNets, the useful messages which intended to the information users may be entirely possible to be eavesdropped by the energy users, which brings huge technical challenges to secure SWIPT in HetNets [19]. Recently, physical-layer security (PLS) has emerged as a prominent technique to guarantee the secrecy communication by exploiting the characteristics of physical wireless channels, while defending against the eavesdroppers [20,21]. Various PLS techniques in SWIPT networks have been studied in MISO and MIMO systems [22-29]. More specifically, Ren et al. [29] studied jointly optimize design of the transmit beamforming and AN of the macrocell base station (MBS) and the femtocell base stations (FBS), the secrecy rate maximization problem of the eavesdropped macrocell users was reformulated by utilizing the one-dimensional line search and successive convex approximation.

In addition, there are some related works integrating the SWIPT in WSNs [30-37]. Guo et al. [30] applied the SWIPT technique to cooperative clustered WSN, a distributed iteration algorithm was proposed by exploiting fractional programming and dual decomposition. Considering different PS abilities of relays in a mobile wireless sensor networks with SWIPT, a resource allocation design (RAD) problem was formulated as a non-convex energy efficiency maximization problem, Guo et al. [31] proposed a cross-layer algorithm for rate control, PS, and power allocation. A unified framework was developed to study the impact of SWIPT on the performance with both time splitting (TS) and power splitting (PS) schemes [32] in a WSN over Nakagami-m fading channels. In the MIMO wireless powered underground sensor network, the optimal RAD problem was considered for throughput the system throughput maximization with quality of service (QoS) assurance in regard to communication 
reliability and diverse data traffic demands [33]. Focusing on two relaying strategies, a downlink (DL) multiple-input single-output (MISO) WSN with the nonorthogonal multiple access technique (NOMA) and SWIPT was investigated over Nakagami-m fading [34], where three antenna-relay-destination selection algorithms were proposed through analysis on downlink (DL) and UL transmissions. Assuming the sensor nodes always work in harvesting-transmitting mode, Hu et al. [35] studied the PLS of uplink (UL) transmission in SWIPT deployed in WSNs, in which the energy/secrecy outage probabilities were derived through comprehensive analysis on DL and UL transmissions, respectively. As for IoT sensor networks with a PS-enabled SWIPT technology, Chae et al. [36] proposed a novel SWIPT strategy where the access point have a choice over either sending a private/common message, and a hybrid beamforming design algorithm was proposed by considering the total transmit power minimization problem. By exploiting a fountain codes in the SWIPT WSNs, Yi et al. [37] proposed a new PS mechanism to adaptively divide data packets into the information data packets and the energy data packets. To the best of our knowledge, secure beamforming design to achieve secrecy capacity, particularly assuming practical power splitting (PS)-based heterogeneous wireless sensor networks (HWSN) with SWIPT transmission, has not been considered previously.

Motivated by the above analysis, in this paper, we consider a SWIPT-enabled two-tier HWSN with MIMO system, where a macrocell sink node and a femtocell sink node transmit co-channel information to the corresponding macrocell sensor nodes (M-SNs), high-priority sensor nodes (HP-SNs) and low-priority sensor nodes (LP-SNs), respectively. In order to harvest more energy from the ambient environment, the HP-SNs can use a power splitter. According to the ambient interference signals, the LP-SNs not only eavesdrop the confidential messages from the M-SNs, but also harvest wireless energy. By applying the interference, an AN-aided secrecy transmission is utilized at the femtocell sink node. In addition, we study the jointly optimization design of the transmit beamforming vectors at the macrocell sink node and femtocell sink node, the AN and PS ratio to maximize the minimal secrecy capacity (MSC) of the wiretapped M-SNs under the secrecy capacity constraint at the HP-SNs, the harvested energy constraint at the HP-SNs and LP-SNs, the transmit power constraint at the macrocell sink node and femtocell sink node. More explicitly, the main contributions of this paper can be summarized as follows:

- Firstly, we establish the system model of a two-tier MIMO HWSN, where SWIPT technique is performed at the femtocell sink node to recharge the HP-SNs and LP-SNs. To be specific, each HP-SN is equipped with power splitter. We consider a general system case where both the M-SNs and HP-SNs are faced under threat of secrecy leakage from the LP-SNs, where each LP-SN act as a malicious user.

- To promote the security performance and harvest more power, the AN is injected designedly into the transmit beamforming at the femtocell sink node. To achieve secrecy enhancement, a joint design problem is formulated for the MSC maximization while considering the fairness among multiple M-SNs. The original problem is non-convex and hard to solve directly.

- In order to reduce the performance loss of the rank relaxation from the SDR technique while solving the non-convex max-min program [38], we apply SCA technique, first-order Taylor series expansion and SPCA method, and then the recast problem can be converted to an SOCP problem [39] which can be directly solved to achieve a near-optimal solution. Moreover, we propose a novel SCA-SPCA-based iterative algorithm, while its convergence property of the proposed algorithm is proved.

Compared with our previous papers [23,24], major additional works and results incorporated in these papers are summarized in the following. (1) This paper has extended the problem of AN power maximization to both perfect and imperfect CSI cases, which introduces substantial changes in the analyses. (2) An SPCA-based iterative algorithm has been proposed to solve the problem such that the computational complexity is largely reduced compared with the 1-D search method used in the previous work. 
The rest of this paper is organized as follows: In Section 2, we presents the HWSN system model and formulates the MSC maximization problem. Section 3 solves the non-convex problem and provide the proposed SCA-SPCA-based iterative algorithm. Section 4 outlines the simulation results. Finally, section 5 concludes this paper.

Notation: Lower-case letters mean scalars, bold-face lower-case letters are denoted by vectors, and boldface upper-case letters are used for matrices. $\mathbb{C}^{M \times L}$ indicates the space of $M \times L$ complex matrices. $|\cdot|$ defines the absolute value of a complex scalar. $\|\mathbf{A}\|_{F}$ describes the Frobenius norm of the matrix $\mathbf{A}, \operatorname{tr}(\mathbf{A})$ indicates the trace of $\mathbf{A} . \mathrm{E}\{\cdot\}$ describes the mathematical expectation. $[x]^{+}$equals $\max \{x, 0\}$.

\section{System Model and Problem Formulation}

In this section, a two-tier downlink HWSN is considered with MIMO-SWIPT, where a femtocell sink node is deployed with a macrocell sink node, as shown in Figure 1. Following [40], the smart antenna technology is applied for the sink nodes. Moreover, the femtocell sink node serves K HP-SNs and $L$ LP-SNs, whereas the macrocell sink node serves $M$ M-SNs. For the sake of notational simplicity, it is assumed that the $m$-th M-SN, the $k$-th HP-SN and the $l$-th LP-SN are denoted by M-SN ${ }_{m}, \mathrm{HP}^{-S_{N}} \mathrm{~N}_{k}$ and $\mathrm{LP}_{-} \mathrm{SN}_{l}$, respectively. The system parameters that we have considered are defined in Table 1 . In the smallcell of this HWSN system, two different types of receivers are considered: (i) one cluster of HP-SNs (such as low-power receivers), denoted by $\mathbb{K}=\left\{\mathrm{HP}-\mathrm{SN}_{1}, \ldots, \mathrm{HP}-\mathrm{SN}_{K}\right\}$, which is located far from the femtocell sink node, (ii) one cluster of LP-SNs (such as sensors), denoted by $\mathbb{L}=\left\{\mathrm{LP}_{-} \mathrm{SN}_{1}, \ldots, \mathrm{LP}-\mathrm{SN} \mathrm{N}_{L}\right\}$, which are located close to the femtocell sink node. It is assumed that each M-SN and HP-SN have single receive antenna, LP-SN is equipped with $N_{E}$ receive antenna, whereas the macrocell sink node and femtocell sink node are equipped with $N_{M} \geq M$ antennas and $N_{F} \geq K+L N_{E}$ transmit antennas, respectively. This system operates as follows: the macrocells share their frequency spectrum with the femtocell to improve the spectral efficiency, whereas the confidential messages from the macrocell sink node and the femtocell sink node may be intercepted by LP-SNs. Additionally, all HP-SNs employ the PS scheme to perform information decoding (ID) and energy harvesting (EH) simultaneously, as shown in Figure 2.

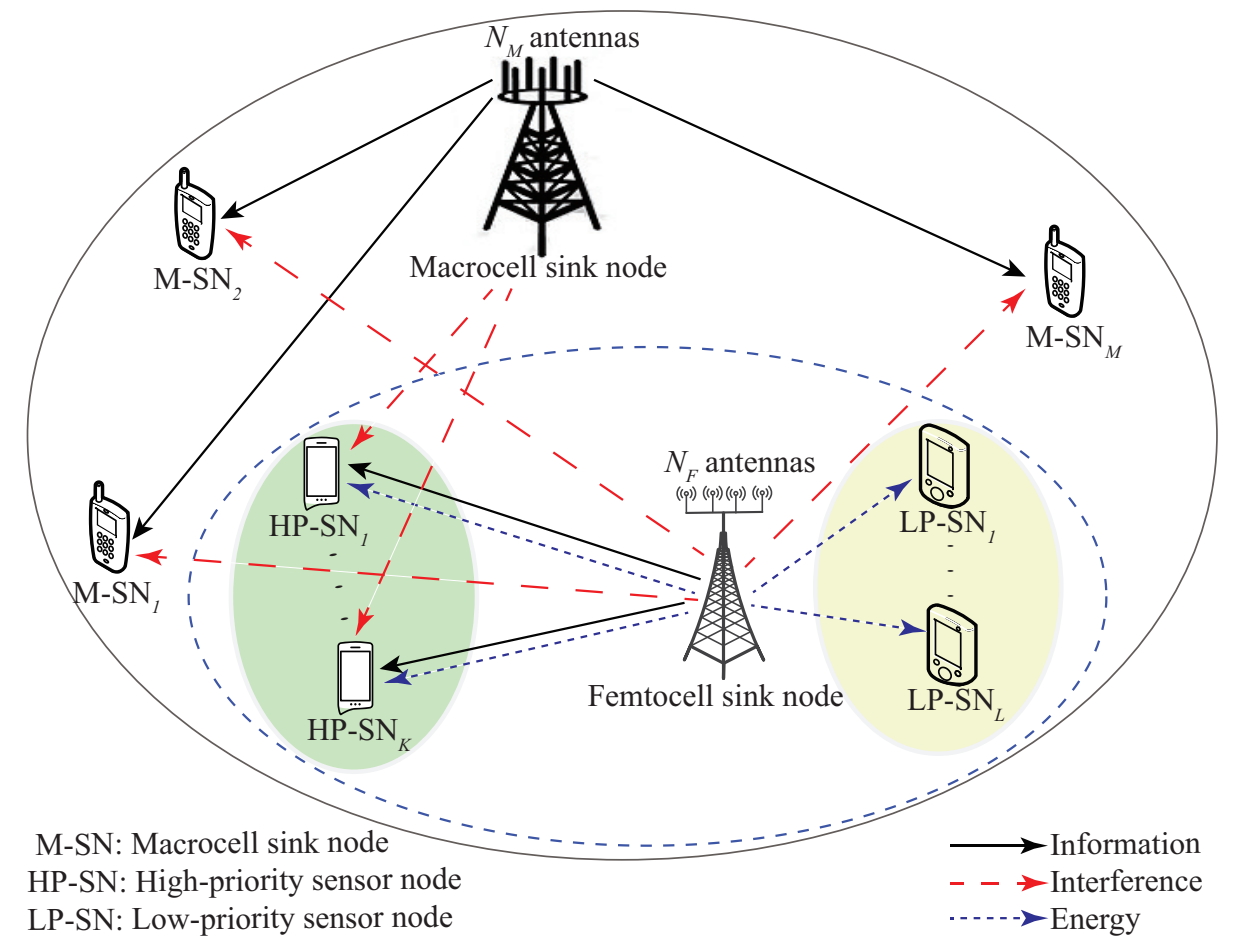

Figure 1. The system model of MIMO Secured HWSN with SWIPT. 


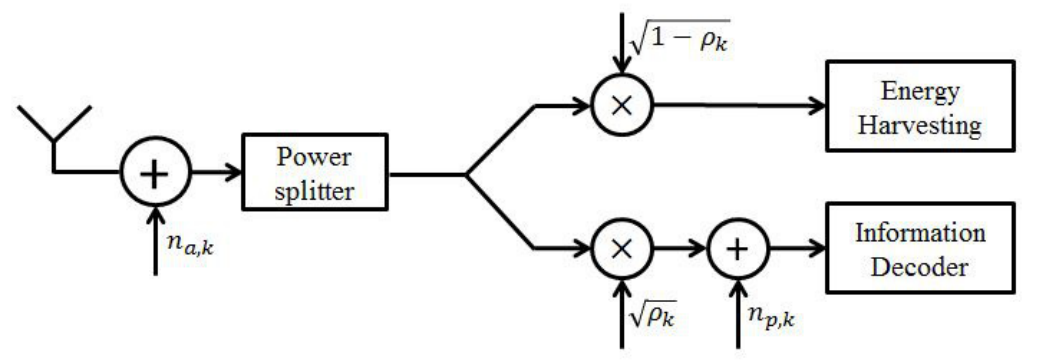

Figure 2. Power splitting scheme in each HP-SN.

Table 1. System Parameters

\begin{tabular}{|c|c|}
\hline Variables & Definition \\
\hline $\mathrm{M}^{-\mathrm{SN}_{m}}$ & the $m$-th M-SN \\
\hline $\mathrm{HP}-\mathrm{SN}_{k}$ & the $k$-th HP-SN \\
\hline $\mathrm{LP}_{-} \mathrm{SN}_{l}$ & the $l$-th LP-SN \\
\hline $\mathbf{s}_{m}$ & the transmit signal from macrocell sink node to $\mathrm{M}-\mathrm{SN}_{m}$ \\
\hline $\mathbf{x}_{S}$ & the transmit signal from femtocell sink node \\
\hline $\mathbf{v}_{m}$ & the transmit beamforming vector from macrocell sink node aiming at $\mathrm{M}-\mathrm{SN}_{m}$ \\
\hline $\mathbf{w}_{k}$ & the transmit beamforming vector from femtocell sink node aiming at $\mathrm{HP}-\mathrm{SN}_{k}$ \\
\hline$s_{p, m}, s_{s, k}$ & the information-bearing signal intended for $\mathrm{M}-\mathrm{SN}_{m}$ and $\mathrm{HP}-\mathrm{SN}_{k}$ \\
\hline $\mathbf{z}$ & the energy-carrying AN \\
\hline $\mathbf{g}_{m}$ & the channel between macrocell sink node and $\mathrm{M}-\mathrm{SN}_{m}$ \\
\hline $\mathbf{g}_{c, k}$ & the channel between macrocell sink node and HP-SN $k$ \\
\hline $\mathbf{G}_{e, l}$ & the channel between macrocell sink node and LP-SN ${ }_{l}^{n}$ \\
\hline $\mathbf{h}_{m}$ & the channel between femtocell sink node and $\mathrm{M}-\mathrm{SN}_{m}$ \\
\hline $\mathbf{h}_{c, k}$ & the channel between femtocell sink node and $\mathrm{HP}-\mathrm{SN}_{k}$ \\
\hline $\mathbf{H}_{e, l}$ & the channel between femtocell sink node and LP-SN ${ }_{l}^{n}$ \\
\hline$n_{m}, n_{c, k}, n_{e, l}$ & the complex Gaussian noise at the M-SN ${ }_{m}$, the HP-SN $k$ and the $\mathrm{LP}^{-\mathrm{SN}_{l}}$ \\
\hline$n_{a, k}$ & additional noise introduced from the RF-to-signal conversion in the ID part at the HP-SN $k$ \\
\hline
\end{tabular}

As an active user in the HWSN, we assume that LP-SN can harvest energy to extend its battery life from the ambient radio frequency (RF) signals. However, LP-SNs may be potential eavesdroppers due to intercepting the information from the femtocell sink node to HP-SNs. Thus, to provide secure transmission in this HWSN, the femtocell sink node applies the AN to transmit beamforming, which not only acts as interference to the LP-SNs, but also supplies energy to the HP-SNs. The secure signal vector $\mathbf{s}$ from the macrocell sink node and $\mathbf{x}_{s}$ from the femtocell sink node can be written as

$$
\begin{aligned}
\mathbf{s} & =\sum_{m=1}^{M} \mathbf{v}_{m} s_{m}, m=1, \ldots, M, \\
\mathbf{x}_{s} & =\sum_{k=1}^{K} \mathbf{w}_{k} s_{s, k}+\mathbf{z}, k=1, \ldots, K,
\end{aligned}
$$

where $\mathbf{v}_{m} \in \mathbb{C}^{N_{P}}$ and $\mathbf{w}_{k} \in \mathbb{C}^{N_{s}}$ define the transmit beamforming vector from the macrocell sink node aiming at M-SN $m$ and from the femtocell sink node aiming at HP-SN ${ }_{k}, s_{m}$ and $s_{s, k}$ are the information-bearing signal intended for ${\mathrm{M}-S N_{m}}_{m}$ and $\mathrm{HP}_{-} \mathrm{SN}_{k}$ with satisfying $\mathbb{E}\left\{s_{m}^{2}\right\}=1$ and $\mathbb{E}\left\{s_{s, k}^{2}\right\}=1$, respectively, and $\mathbf{z}$ represents the energy-carrying AN. 
Therefore, the received signal at the $\mathrm{M}-\mathrm{SN}_{m}$, the $\mathrm{HP}-\mathrm{SN}_{k}$, and the $\mathrm{LP}^{-\mathrm{SN}_{l}}$ can be given by, respectively,

$$
\begin{aligned}
& y_{m}=\underbrace{\mathbf{g}_{m}^{H} \mathbf{v}_{m} s_{m}}_{\text {desired signal }}+\underbrace{\mathbf{g}_{m}^{H} \sum_{i=1, i \neq m}^{M}\left(\mathbf{v}_{i} s_{p, i}\right)}_{\text {intra-tier interference }}+\underbrace{\mathbf{h}_{m}^{H}\left(\sum_{k=1}^{K} \mathbf{w}_{k} s_{s, k}+\mathbf{z}\right)}_{\text {inter-tier interference }}+n_{m}, m=1, \ldots, M, \\
& y_{c, k}=\underbrace{\mathbf{h}_{c, k}^{H} \mathbf{w}_{k} s_{s, k}}_{\text {desired signal }}+\underbrace{\mathbf{h}_{c, k}^{H} \mathbf{z}+\mathbf{h}_{c, k}^{H} \sum_{i=1, i \neq k}^{K}\left(\mathbf{w}_{i} s_{s, i}\right)}_{\text {intra-tier interference }}+\underbrace{\mathbf{g}_{c, k}^{H} \sum_{m=1}^{M} \mathbf{v}_{m} s_{m}}_{\text {inter-tier interference }}+n_{c, k}, k=1, \ldots, K, \\
& \mathbf{y}_{e, l}=\underbrace{\mathbf{H}_{e, l}^{H} \mathbf{w}_{l} s_{s, l}}_{\text {desired signal }}+\underbrace{\mathbf{H}_{e, l}^{H} \mathbf{z}+\mathbf{H}_{e, l}^{H} \sum_{i=1, i \neq l}^{K}\left(\mathbf{w}_{i} s_{s, i}\right)}_{\text {intra-tier interference }}+\underbrace{\mathbf{G}_{e, l}^{H} \sum_{m=1}^{M} \mathbf{v}_{m} s_{m}}_{\text {inter-tier interference }}+\mathbf{n}_{e, l}, l=1, \ldots, L,
\end{aligned}
$$

where $\mathbf{g}_{m} \in \mathbb{C}$ and $\mathbf{h}_{m} \in \mathbb{C}^{N_{S}}$ are denoted by the channel between macrocell sink node and M-SN $\mathrm{N}_{m}$ as well as that between femtocell sink node and M-SN ${ }_{m}, \mathbf{g}_{c, k} \in \mathbb{C}^{N_{P} \times N_{C}}$ and $\mathbf{h}_{c, k} \in \mathbb{C}^{N_{S} \times N_{C}}$ indicate the channel vectors between macrocell sink node and HP-SN ${ }_{k}$ as well as that between the femtocell sink node and the HP-SN $N_{k}, \mathbf{G}_{e, l} \in \mathbb{C}^{N_{P} \times N_{E}}$ and $\mathbf{H}_{e, l} \in \mathbb{C}^{N_{S} \times N_{E}}$ are the channel vectors between the macrocell sink node and LP-SN $l$ as well as that between the femtocell sink node and LP-SN ${ }_{l}, s_{m}$ is confidential information-bearing signal for $\mathrm{M}-\mathrm{SN}_{m}$ from the macrocell sink node satisfying $\mathbb{E}\left\{s_{m}^{2}\right\}=1$. In addition, $n_{m} \sim \mathcal{C N}\left(0, \sigma_{m}^{2}\right), n_{a, k} \sim \mathcal{C N}\left(0, \sigma_{a, k}^{2}\right)$ and $\mathbf{n}_{e, l} \sim \mathcal{C N}\left(0, \sigma_{e, l}^{2} \mathbf{I}\right)$ denote the complex AWGN at the $\mathrm{M}-\mathrm{SN}_{m}$, the HP-SN $\mathrm{H}_{k}$ and the LP-SN ${ }_{l}$, respectively.

Since the HP-SN utilizes PS scheme to simultaneously implement ID and EH, the received signal at the HP-SN ${ }_{k}$ is divided into ID portion and EH portion by the PS ratio $\rho_{k} \in(0,1]$. Hence, the split signal for ID part and $\mathrm{EH}$ part at the $\mathrm{HP}-\mathrm{SN}_{k}$ can be expressed as, respectively,

$$
\begin{aligned}
& y_{c, k}^{I D}=\sqrt{\rho_{k}}\left(\mathbf{h}_{c, k}^{H} \mathbf{w}_{k} s_{s, k}+\mathbf{h}_{c, k}^{H} \mathbf{z}+\mathbf{h}_{c, k}^{H} \sum_{i=1, i \neq k}^{K}\left(\mathbf{w}_{i} s_{s, i}\right)+\mathbf{g}_{c, k}^{H} \sum_{m=1}^{M} \mathbf{v}_{m} s_{m}+n_{a, k}\right)+n_{p, k}, \\
& y_{c, k}^{E H}=\sqrt{1-\rho_{k}}\left(\mathbf{h}_{c, k}^{H} \mathbf{w}_{k} s_{s, k}+\mathbf{h}_{c, k}^{H} \mathbf{z}+\mathbf{h}_{c, k}^{H} \sum_{i=1, i \neq k}^{K}\left(\mathbf{w}_{i} s_{s, i}\right)+\mathbf{g}_{c, k}^{H} \sum_{m=1}^{M} \mathbf{v}_{m} s_{m}+n_{a, k}\right),
\end{aligned}
$$

where $n_{p, k} \sim \mathcal{C N}\left(0, \delta_{p, k}^{2}\right)$ is the additional Gaussian noise resulted in the RF-to-signal conversion for the ID part at the HP-SN ${ }_{k}$.

Thus, the channel capacity of the HP-SN $k$ is expressed as

$$
R_{c, k}=\log \left(1+\frac{\rho_{k} \mathbf{h}_{c, k}^{H} \mathbf{w}_{k} \mathbf{w}_{k}^{H} \mathbf{h}_{c, k}}{\rho_{k}\left(\mathbf{h}_{c, k}^{H}\left(\sum_{j \neq k} \mathbf{w}_{j} \mathbf{w}_{j}^{H}+\mathbf{z z}^{H}\right) \mathbf{h}_{c, k}+\mathbf{g}_{c, k}^{H} \sum_{m=1}^{M}\left(\mathbf{v}_{m} \mathbf{v}_{m}^{H}\right) \mathbf{g}_{c, k}+\sigma_{a, k}^{2}\right)+\delta_{p, k}^{2}}\right) .
$$

The channel capacity of the LP-SN $N_{l}$ for decoding the desired signal of the HP-SN $\mathrm{H}_{k}$ can be represented as

$$
R_{c, l k}=\log \left|\mathbf{I}+\left(\sigma_{e, l}^{2} \mathbf{I}+\left(\mathbf{H}_{e, l}^{H}\left(\sum_{j \neq k} \mathbf{w}_{j} \mathbf{w}_{j}^{H}+\mathbf{z z}^{H}\right) \mathbf{H}_{e, l}+\mathbf{G}_{e, l}^{H} \sum_{m=1}^{M}\left(\mathbf{v}_{m} \mathbf{v}_{m}^{H}\right) \mathbf{G}_{e, l}\right)\right)^{-1} \mathbf{H}_{e, l}^{H} \mathbf{w}_{k} \mathbf{w}_{k}^{H} \mathbf{H}_{e, l}\right| .
$$

Thus, the secrecy capacity of the HP-SN $\mathrm{S}_{k}$ can be written as

$$
C_{c, k}=\left[R_{c, k}-\max _{l} R_{c, l k}\right]^{+}, \forall k, l .
$$


Additionally, the channel capacity of the $\mathrm{M}_{-} \mathrm{SN}_{m}$ can be expressed as

$$
R_{m}=\log \left(1+\frac{\mathbf{g}_{m}^{H} \mathbf{v}_{m} \mathbf{v}_{m}^{H} \mathbf{g}_{m}}{\mathbf{h}_{m}^{H}\left(\sum_{j=1}^{K} \mathbf{w}_{j} \mathbf{w}_{j}^{H}+\mathbf{z z}^{H}\right) \mathbf{h}_{m}+\sigma_{m}^{2}+\mathbf{g}_{m}^{H} \sum_{j \neq m}^{M}\left(\mathbf{v}_{j} \mathbf{v}_{j}^{H}\right) \mathbf{g}_{m}}\right) .
$$

Suppose that the $\mathrm{LP}_{-} \mathrm{SN}_{l}$ is an eavesdropper to decode the message for the M-SN $m$. The channel capacity of the $\mathrm{LP}_{-} \mathrm{SN}_{l}$ for decoding the $\mathrm{M}-\mathrm{SN}_{m}$ is given as

$$
R_{m, l}=\log \left|\mathbf{I}+\left(\mathbf{H}_{e, l}^{H}\left(\sum_{j=1}^{K} \mathbf{w}_{j} \mathbf{w}_{j}^{H}+\mathbf{z z} \mathbf{z}^{H}\right) \mathbf{H}_{e, l}+\mathbf{G}_{e, l}^{H} \sum_{j \neq m}\left(\mathbf{v}_{j} \mathbf{v}_{j}^{H}\right) \mathbf{G}_{e, l}+\sigma_{e, l}^{2} \mathbf{I}\right)^{-1} \mathbf{G}_{e, l}^{H} \mathbf{v}_{m} \mathbf{v}_{m}^{H} \mathbf{G}_{e, l}\right| .
$$

Hence, following the concept of secrecy capacity in [20-22], the secrecy capacity of the M-SN $m$ can be written as

$$
C_{m}=\left[R_{m}-\max _{l} R_{m, l}\right]^{+}, \forall l .
$$

where $[a]^{+}$indicates the $\max (0, a)$.

Moreover, the HP-SNs employs the PS ratio to harvest the power carried from the RF signal. In order to harvest the carried energy, the LP-SN receiver does not need to transform the received signal to the baseband. However, due to the law of energy conservation, we assume that the total harvested energy at the HP-SN ${ }_{k}$ and the LP-SN ${ }_{l}$, i.e., the RF-band power normalized by the baseband symbol, denoted by $E_{c, k}$ and $E_{e, l}$, respectively, from all $N_{E}$ receive antennas at the LP-SN receiver is proportional to that of the received RF baseband signal, i.e.,

$$
\begin{aligned}
& E_{c, k}=\eta_{c, k}\left(1-\rho_{k}\right)\left(\mathbf{h}_{c, k}^{H}\left(\sum_{j=1}^{K} \mathbf{w}_{j} \mathbf{w}_{j}^{H}+\mathbf{z z}^{H}\right) \mathbf{h}_{c, k}+\mathbf{g}_{c, k}^{H} \sum_{m=1}^{M}\left(\mathbf{v}_{m} \mathbf{v}_{m}^{H}\right) \mathbf{g}_{c, k}+\sigma_{a, k}^{2}\right), \forall k, \\
& E_{e, l}=\eta_{e, l}\left(\operatorname{tr}\left(\mathbf{H}_{e, l}^{H}\left(\sum_{j=1}^{K} \mathbf{w}_{j} \mathbf{w}_{j}^{H}+\mathbf{z z}^{H}\right) \mathbf{H}_{e, l}\right)+\operatorname{tr}\left(\mathbf{G}_{e, l}^{H} \sum_{m=1}^{M}\left(\mathbf{v}_{m} \mathbf{v}_{m}^{H}\right) \mathbf{G}_{e, l}\right)+N_{E} \sigma_{e, l}^{2}\right), \forall l,
\end{aligned}
$$

where $0 \leq \eta_{c, k} \leq 1$ and $0 \leq \eta_{e, l} \leq 1$ denote the energy conversion efficiency at the HP-SN ${ }_{k}$ and LP-SN ${ }_{l}$, respectively.

In this paper, our objective is to secure the WIT to legitimated users (i.e., M-SNs and HP-SNs) in the presence of potential eavesdroppers (i.e., LP-SNs) by jointly designing the AN-aided transmit beamforming. Moverover, we aim to promote the MSC performance by guaranteeing all M-SNs can achieve a certain secrecy capacity level. To this end, we maximize the MSC of the M-SN $m$ under the secrecy capacity constraint at the HP-SNs, the harvested energy constraint at the HP-SNs and LP-SNs, as well as the transmit power constraint at the macrocell sink node and femtocell sink node. Based on the system models, the MSC maximization (MSCM) problem is formulated as

$$
\begin{aligned}
\max _{\mathbf{w}_{k}, \mathbf{z}, \mathbf{v}_{m}, \rho_{k}} & \min _{m=1, \ldots, M} C_{m} \\
\text { s.t. } \quad & \min _{k} C_{c, k} \geq \bar{R}^{c}, \forall k, \\
& \min _{k} E_{c, k} \geq \bar{E}^{c}, \forall k, \\
& \min _{l} E_{e, l} \geq \bar{E}^{e}, \forall l, \\
& \sum_{k=1}^{K}\left\|\mathbf{w}_{k}\right\|^{2}+\|\mathbf{z}\|^{2} \leq P, \forall k, \sum_{k=1}^{K}\left\|\mathbf{w}_{k}\right\|^{2} \leq P_{1}, \sum_{m=1}^{M}\left\|\mathbf{v}_{m}\right\|^{2} \leq P_{2}, \\
& 0 \leq \rho_{k} \leq 1,
\end{aligned}
$$


where $\bar{R}^{c}$ denotes the secrecy capacity requirement of the HP-SN$k, \bar{E}^{c}$ and $\bar{E}^{e}$ mean the harvested power requirement of the HP-SN ${ }_{k}$ and the $\mathrm{LP}_{-} \mathrm{SN}_{l}$, respectively, $P$ represents the total transmit power at the femtocell sink node, $P_{1}$ indicates the transmit power about the information signal part at the femtocell sink node, $P_{2}$ stands for the total transmit power at the macrocell sink node. The constraints (8a) guarantees that the minimum secrecy rate should be achieved at the HP-SN${ }_{k}$. The constraints and (8b) and (8c) guarantee that the minimum harvested power at the HP-SN $k$ and the LP-SN ${ }_{l}$ are no less than $\bar{E}^{c}$ and $\bar{E}^{e}$, respectively. The constraint (8d) limits the transmit power. Obviously, it is seen that problem (8) is non-convex due to the existence of coupling among variables $\rho_{k}$ and $\mathbf{w}_{k}$, which is difficult to solved directly.

\section{Proposed Algorithm}

In this section, we will propose an iterative algorithm to obtain near-optimal solution by applying the SCA method and SPCA technology [41]. To tackle problem (8) we firstly introduce an equivalent reformulation about problem (8). To circumvent this issue, problem (8) can be rewritten as

$$
\begin{aligned}
& \max _{\mathbf{w}_{k}, \mathbf{z}, \mathbf{v}_{m}, \rho_{k}} \min _{m=1, \ldots, M} \log \left(1+\frac{\mathbf{g}_{m}^{H} \mathbf{v}_{m} \mathbf{v}_{m}^{H} \mathbf{g}_{m}}{\mathbf{h}_{m}^{H}\left(\sum_{k=1}^{K} \mathbf{w}_{k} \mathbf{w}_{k}^{H}+\mathbf{z z}^{H}\right) \mathbf{h}_{m}+\mathbf{g}_{m}^{H} \sum_{j \neq m}\left(\mathbf{v}_{j} \mathbf{v}_{j}^{H}\right) \mathbf{g}_{m}+\sigma_{m}^{2}}\right) \\
& -\log \left|\mathbf{I}+\left(\mathbf{H}_{e, l}^{H}\left(\sum_{k=1}^{K} \mathbf{w}_{k} \mathbf{w}_{k}^{H}+\mathbf{z z} \mathbf{z}^{H}\right) \mathbf{H}_{e, l}+\mathbf{G}_{e, l}^{H} \sum_{j \neq m}\left(\mathbf{v}_{j} \mathbf{v}_{j}^{H}\right) \mathbf{G}_{e, l}+\sigma_{e, l}^{2} \mathbf{I}\right)^{-1} \mathbf{G}_{e, l}^{H} \mathbf{v}_{m} \mathbf{v}_{m}^{H} \mathbf{G}_{e, l}\right| \\
& \text { s.t. } \log \left(1+\frac{\rho_{k} \mathbf{h}_{c, k}^{H} \mathbf{w}_{k} \mathbf{w}_{k}^{H} \mathbf{h}_{c, k}}{\rho_{k}\left(\mathbf{h}_{c, k}^{H}\left(\sum_{j \neq k} \mathbf{w}_{j} \mathbf{w}_{j}^{H}+\mathbf{z z}^{H}\right) \mathbf{h}_{c, k}+\mathbf{g}_{c, k}^{H} \sum_{m=1}^{M}\left(\mathbf{v}_{m} \mathbf{v}_{m}^{H}\right) \mathbf{g}_{c, k}+\sigma_{a, k}^{2}\right)+\delta_{p, k}^{2}}\right) \\
& -\log \left|\mathbf{I}+\left(\sigma_{e, l}^{2} \mathbf{I}+\mathbf{H}_{e, l}^{H}\left(\sum_{j \neq k} \mathbf{w}_{j} \mathbf{w}_{j}^{H}+\mathbf{z z}^{H}\right) \mathbf{H}_{e, l}+\mathbf{G}_{e, l}^{H} \sum_{m=1}^{M}\left(\mathbf{v}_{m} \mathbf{v}_{m}^{H}\right) \mathbf{G}_{e, l}\right)^{-1} \mathbf{H}_{e, l}^{H} \mathbf{w}_{k} \mathbf{w}_{k}^{H} \mathbf{H}_{e, l}\right| \geq \bar{R}^{c}, \\
& \eta_{k}\left(1-\rho_{k}\right)\left(\operatorname{tr}\left(\mathbf{h}_{c, k}^{H}\left(\sum_{j=1}^{K} \mathbf{w}_{j} \mathbf{w}_{j}^{H}+\mathbf{z z} \mathbf{z}^{H}\right) \mathbf{h}_{c, k}\right)+\operatorname{tr}\left(\mathbf{g}_{c, k}^{H} \sum_{m=1}^{M}\left(\mathbf{v}_{m} \mathbf{v}_{m}^{H}\right) \mathbf{g}_{c, k}\right)+N_{C} \sigma_{a, k}^{2}\right) \geq \bar{E}^{c}, \forall k, \\
& \eta_{l}\left(\operatorname{tr}\left(\mathbf{H}_{e, l}^{H}\left(\sum_{j=1}^{K} \mathbf{w}_{j} \mathbf{w}_{j}^{H}+\mathbf{z z} \mathbf{z}^{H}\right) \mathbf{H}_{e, l}\right)+\operatorname{tr}\left(\mathbf{G}_{e, l}^{H} \sum_{m=1}^{M}\left(\mathbf{v}_{m} \mathbf{v}_{m}^{H}\right) \mathbf{G}_{e, l}\right)+\sigma_{e, l}^{2}\right) \geq \bar{E}^{e}, \forall l, \\
& \text { (8d), (8e). }
\end{aligned}
$$

It is observed that the determinant functions is in the $R_{c, l k}$ of the objective function (9a). We consider the following Lemma 1, which will be applied to provide a convex approximation form to $C_{l, k}$.

Lemma 1. ([42]) For any matrix $\boldsymbol{A}$, which $\boldsymbol{A}$ is positive semi-definite, i.e., $\boldsymbol{A} \succeq \boldsymbol{0}$. It holds true that

$$
|\boldsymbol{I}+\boldsymbol{A}| \geq 1+\operatorname{tr}(\boldsymbol{A}),
$$

where the equality holds if and only if $\operatorname{rank}(\boldsymbol{A}) \leq 1$.

Proof. See Appendix A.

We introduce two real-valued slack variables $\gamma_{c}$ and $\gamma_{e}$. By applying the Lemma 1, problem (9) can be rewritten as 


$$
\begin{aligned}
& \max _{\mathbf{w}_{k}, \mathbf{z}, \mathbf{v}_{m}, \rho_{k}, \gamma_{c}, \gamma_{e}} \gamma_{c}-\gamma_{e} \\
& \text { s.t. } \frac{\mathbf{g}_{m}^{H} \mathbf{v}_{m} \mathbf{v}_{m}^{H} \mathbf{g}_{m}}{\mathbf{h}_{m}^{H}\left(\sum_{k=1}^{K} \mathbf{w}_{k} \mathbf{w}_{k}^{H}+\mathbf{z} \mathbf{z}^{H}\right) \mathbf{h}_{m}+\mathbf{g}_{m}^{H} \sum_{j \neq m}\left(\mathbf{v}_{j} \mathbf{v}_{j}^{H}\right) \mathbf{g}_{m}+\sigma_{m}^{2}} \geq 2^{\gamma_{c}}-1 \text {, } \\
& \frac{\operatorname{tr}\left(\mathbf{G}_{e, l}^{H} \mathbf{v}_{m} \mathbf{v}_{m}^{H} \mathbf{G}_{e, l}\right)}{\operatorname{tr}\left(\mathbf{H}_{e, l}^{H}\left(\sum_{k=1}^{K} \mathbf{w}_{k} \mathbf{w}_{k}^{H}+\mathbf{z z}^{H}\right) \mathbf{H}_{e, l}\right)+\mathbf{G}_{e, l}^{H} \sum_{j \neq m}\left(\mathbf{v}_{j} \mathbf{v}_{j}^{H}\right) \mathbf{G}_{e, l}+\sigma_{e, l}^{2}} \leq 2^{\gamma_{e}}-1, \\
& \text { (9b), (9c), (9d), (8d), (8e). }
\end{aligned}
$$

In order to make the problem (11) tractable, by introducing the auxiliary variables $v_{m}, w_{m}, u_{m}$, $t_{l}, e_{l}$, and $q_{l}$, we define new matrices $\mathbf{G}_{m}=\mathbf{g}_{m} \mathbf{g}_{m}^{H}, \mathbf{H}_{m}=\mathbf{h}_{m} \mathbf{h}_{m}^{H}, \mathbf{G}_{c, k}=\mathbf{g}_{c, k} \mathbf{g}_{c, k}^{H} \mathbf{H}_{c, k}=\mathbf{h}_{c, k} \mathbf{h}_{c, k^{\prime}}^{H}$ $\overline{\mathbf{G}}_{e, l}=\mathbf{G}_{e, l} \mathbf{G}_{e, l}^{H}$, and $\overline{\mathbf{H}}_{e, l}=\mathbf{H}_{e, l} \mathbf{H}_{e, l}^{H}$. The constraints (11b) and (11c) can be written as, respectively,

$$
\begin{aligned}
& \mathbf{v}_{m}^{H} \mathbf{G}_{m} \mathbf{v}_{m} \geq e^{v_{m}}, \\
& e^{w_{m}} \geq \sum_{k=1}^{K} \mathbf{w}_{k}^{H} \mathbf{H}_{m} \mathbf{w}_{k}+\mathbf{z}^{H} \mathbf{H}_{m} \mathbf{z}+\sum_{j \neq m}\left(\mathbf{v}_{j}^{H} \mathbf{G}_{m} \mathbf{v}_{j}\right)+\sigma_{m}^{2}, \\
& e^{u_{m}} \geq 2^{\gamma_{c}}-1, \\
& v_{m}-w_{m} \geq u_{m} .
\end{aligned}
$$

$$
\begin{aligned}
& \mathbf{v}_{m}^{H} \overline{\mathbf{G}}_{e, l} \mathbf{v}_{m} \leq e^{t_{l}}, \forall m, \forall l, \\
& e^{e_{l}} \leq \sum_{k=1}^{K} \mathbf{w}_{k}^{H} \overline{\mathbf{H}}_{e, l} \mathbf{w}_{k}+\mathbf{z}^{H} \overline{\mathbf{H}}_{e, l} \mathbf{z}+\sum_{j \neq m}\left(\mathbf{v}_{j}^{H} \overline{\mathbf{G}}_{e, l} \mathbf{v}_{j}\right)+\sigma_{e, l}^{2}, \\
& e^{q_{l}} \leq 2^{\gamma_{e}}-1, \\
& t_{l}-e_{l} \leq q_{l} .
\end{aligned}
$$

According to [43], it is well-known that a constraint is such that a concave function is greater than or equal to a convex function, then the constraint is a convex form. Therefore, it is easily seen that (12a) and (13b) are convex now. However, (12b), (12c), (13a) and (13c) are still non-convex. Nevertheless, the functions $e^{w_{m}}$ and $e^{u_{m}}$ on the right-hand side (RHS) of (12b) and (12c) are convex, which makes (12) easier to deal with than (11b). Similarly, the functions $e^{t_{l}}$ and $2^{\gamma_{e}}-1$ on the left-hand sides (LHS) of (13a) and (13c) are also convex, we obtain that (13) is easier to handle than (11c).

Following the idea of $[23,44]$, we apply the SCA method to deal with (12b), (12c), (13a) and (13c). To this end, let us define $\bar{w}_{m}^{(n)}, \bar{u}_{m}^{(n)}, \bar{t}_{l}^{(n)}$ and $\bar{\gamma}_{e}^{(n)}$ as the variables $\bar{w}_{m}, \bar{u}_{m}, \bar{t}_{l}$ and $\bar{\gamma}_{e}$ at the $n$-th iteration for a novel SCA-SPCA-based iterative algorithm given below. By employing first-order Taylor series expansions on $e^{w_{m}}, e^{u_{m}}, e^{t_{l}}$ and $2^{\gamma_{e}}$ i.e., $e^{\bar{w}_{m}^{(n)}}\left(w_{m}-\bar{w}_{m}^{(n)}+1\right), e^{\bar{u}_{m}^{(n)}}\left(u_{m}-\bar{u}_{m}^{(n)}+1\right), e^{\bar{t}_{l}^{(n)}}\left(t_{l}-\bar{t}_{l}^{(n)}+1\right)$ and $2^{(n)}\left(\left(\gamma_{e}-\bar{\gamma}_{e}^{(n)}\right) \ln 2+1\right)$, the linearization of the non-convex constraints in (12b), (12c), (13a) and (13c) are given by

$$
\begin{aligned}
& e^{\bar{w}_{m}^{(n)}}\left(w_{m}-\bar{w}_{m}^{(n)}+1\right) \geq \sum_{k=1}^{K} \mathbf{w}_{k}^{H} \mathbf{H}_{m} \mathbf{w}_{k}+\mathbf{z}^{H} \mathbf{H}_{m} \mathbf{z}+\sum_{j \neq m}\left(\mathbf{v}_{j}^{H} \mathbf{G}_{m} \mathbf{v}_{j}\right)+\sigma_{m}^{2}, \\
& e^{\bar{u}_{m}^{(n)}}\left(u_{m}-\bar{u}_{m}^{(n)}+1\right) \geq 2^{\gamma_{c}}-1, \\
& e^{\bar{t}_{l}^{(n)}}\left(t_{l}-\bar{t}_{l}^{(n)}+1\right) \geq \mathbf{v}_{m}^{H} \overline{\mathbf{G}}_{e, l} \mathbf{v}_{m}, \forall m, \forall l \\
& 2^{\bar{\gamma}_{e}^{(n)}}\left(\left(\gamma_{e}-\bar{\gamma}_{e}^{(n)}\right) \ln 2+1\right)-1 \geq e^{q_{l}} .
\end{aligned}
$$


In addition, for the constraint (9b), we will reformulate it by using SPCA method. By introducing two slack variables $s_{1}>0$ and $s_{2}>0,(9 b)$ can be recast as

$$
\begin{aligned}
& \log \left(s_{1} s_{2}\right) \geq \bar{R}^{c}, \forall l, \\
& 1+\frac{\rho_{k} \mathbf{h}_{c, k}^{H} \mathbf{w}_{k} \mathbf{w}_{k}^{H} \mathbf{h}_{c, k}}{\rho_{k}\left(\mathbf{h}_{c, k}^{H}\left(\sum_{j \neq k} \mathbf{w}_{j} \mathbf{w}_{j}^{H}+\mathbf{z z}^{H}\right) \mathbf{h}_{c, k}+\mathbf{g}_{c, k}^{H} \sum_{m=1}^{M}\left(\mathbf{v}_{m} \mathbf{v}_{m}^{H}\right) \mathbf{g}_{c, k}+\sigma_{a, k}^{2}\right)+\delta_{p, k}^{2}} \geq s_{1}, \\
& \left|\mathbf{I}+\left(\sigma_{e, l}^{2} \mathbf{I}+\mathbf{H}_{e, l}^{H}\left(\sum_{j \neq k} \mathbf{w}_{j} \mathbf{w}_{j}^{H}+\mathbf{z z}^{H}\right) \mathbf{H}_{e, l}+\mathbf{G}_{e, l}^{H} \sum_{m=1}^{M}\left(\mathbf{v}_{m} \mathbf{v}_{m}^{H}\right) \mathbf{G}_{e, l}\right)^{-1} \mathbf{H}_{e, l}^{H} \mathbf{w}_{k} \mathbf{w}_{k}^{H} \mathbf{H}_{e, l}\right| \leq \frac{1}{s_{2}} .
\end{aligned}
$$

By apply the Lemma 1, the constraint (15c) can be recast, respectively, as

$$
1+\frac{\operatorname{tr}\left(\mathbf{H}_{e, l}^{H} \mathbf{w}_{k} \mathbf{w}_{k}^{H} \mathbf{H}_{e, l}\right)}{\sigma_{e, l}^{2}+\operatorname{tr}\left(\mathbf{H}_{e, l}^{H}\left(\sum_{j \neq k} \mathbf{w}_{j} \mathbf{w}_{j}^{H}+\mathbf{z z} \mathbf{z}^{H}\right) \mathbf{H}_{e, l}+\mathbf{G}_{e, l}^{H} \sum_{m=1}^{M}\left(\mathbf{v}_{m} \mathbf{v}_{m}^{H}\right) \mathbf{G}_{e, l}\right)} \leq \frac{1}{s_{2}} .
$$

Then, (15) can be further simplified as

$$
\begin{aligned}
& s_{1} s_{2} \geq 2^{\bar{R}^{c}}, \forall l, \\
& \frac{\mathbf{h}_{c, k}^{H} \mathbf{w}_{k} \mathbf{w}_{k}^{H} \mathbf{h}_{c, k}}{\mathbf{h}_{c, k}^{H}\left(\sum_{j \neq k} \mathbf{w}_{j} \mathbf{w}_{j}^{H}+\mathbf{z z}^{H}\right) \mathbf{h}_{c, k}+\mathbf{g}_{c, k}^{H} \sum_{m=1}^{M}\left(\mathbf{v}_{m} \mathbf{v}_{m}^{H}\right) \mathbf{g}_{c, k}+\sigma_{a, k}^{2}+\frac{\delta_{p, k}^{2}}{\rho_{k}}} \geq s_{1}-1, \\
& \frac{\sigma_{e, l}^{2}+\operatorname{tr}\left(\mathbf{H}_{e, l}^{H}\left(\sum_{j \neq k} \mathbf{w}_{j} \mathbf{w}_{j}^{H}+\mathbf{z z}^{H}\right) \mathbf{H}_{e, l}+\mathbf{G}_{e, l}^{H} \sum_{m=1}^{M}\left(\mathbf{v}_{m} \mathbf{v}_{m}^{H}\right) \mathbf{G}_{e, l}\right)}{\sigma_{e, l}^{2}+\operatorname{tr}\left(\mathbf{H}_{e, l}^{H}\left(\sum_{j=1}^{K} \mathbf{w}_{j} \mathbf{w}_{j}^{H}+\mathbf{z z}^{H}\right) \mathbf{H}_{e, l}+\mathbf{G}_{e, l}^{H} \sum_{m=1}^{M}\left(\mathbf{v}_{m} \mathbf{v}_{m}^{H}\right) \mathbf{G}_{e, l}\right)} \geq s_{2} .
\end{aligned}
$$

The inequality constraint (17a) is equivalent to $2^{\bar{R}^{c}+2}+\left(s_{1}-s_{2}\right)^{2} \leq\left(s_{1}+s_{2}\right)^{2}$. Then, we can transform it to a conic quadratic-representable function as (18)

$$
\left\|\left[\sqrt{2^{\bar{R}^{c}+2}} s_{1}-s_{2}\right]\right\| \leq s_{1}+s_{2}, \forall l .
$$

And then, we transform the inequality constraints (17b) and (17c) into the following form as

$$
\begin{aligned}
& \sum_{j \neq k} \mathbf{w}_{j}^{H} \mathbf{H}_{c, k} \mathbf{w}_{j}+\mathbf{z}^{H} \mathbf{H}_{c, k} \mathbf{z}+\sum_{m=1}^{M} \mathbf{v}_{m}^{H} \mathbf{G}_{c, k} \mathbf{v}_{m}+\sigma_{a, k}^{2}+\frac{\delta_{p, k}^{2}}{\rho_{k}} \leq \frac{\mathbf{w}_{k}^{H} \mathbf{H}_{c, k} \mathbf{w}_{k}}{s_{1}-1}, \\
& \sigma_{e, l}^{2}+\sum_{j=1}^{K} \mathbf{w}_{j}^{H} \overline{\mathbf{H}}_{e, l} \mathbf{w}_{j}+\mathbf{z}^{H} \overline{\mathbf{H}}_{e, l} \mathbf{z}+\sum_{m=1}^{M} \mathbf{v}_{m}^{H} \overline{\mathbf{G}}_{e, l} \mathbf{v}_{m} \\
& \leq \frac{\sigma_{e, l}^{2}+\sum_{j \neq k} \mathbf{w}_{j}^{H} \overline{\mathbf{H}}_{e, l} \mathbf{w}_{j}+\mathbf{z}^{H} \overline{\mathbf{H}}_{e, l} \mathbf{z}+\sum_{m=1}^{M} \mathbf{v}_{m}^{H} \overline{\mathbf{G}}_{e, l} \mathbf{v}_{m}}{s_{2}}
\end{aligned}
$$

It is observed that the constraints (19a) and (19b) are non-convex. Fortunately, the RHS of both constraints is the quadratic-over-linear (QoL) function, which are convex form [43]. According to the idea of the constrained convex procedure [45], these QoL functions can be replaced by their first-order Taylor expansions, which can be converted into a convex programming problem. Specifically, we firstly define a function as

$$
f_{\mathbf{B}, b}(\mathbf{w}, y)=\frac{\mathbf{u}^{H} \mathbf{B u}}{y-b}
$$


where $y \geq b$ and $\mathbf{B} \succeq \mathbf{0}$. Then, the first-order Taylor expansion of (20) at a certain point $(\tilde{\mathbf{u}}, \tilde{b})$ is written by

$$
F_{\mathbf{B}, b}(\mathbf{u}, y, \tilde{\mathbf{u}}, \tilde{y})=\frac{2 \Re\left\{\tilde{\mathbf{u}}^{H} \mathbf{B u}\right\}}{\tilde{y}-b}-\frac{\tilde{\mathbf{u}}^{H} \mathbf{B} \tilde{\mathbf{u}}}{(\tilde{y}-b)^{2}}(y-b)
$$

By utilizing the above results of first-order Taylor expansion, for the certain points $\left(\mathbf{w}_{k}, \tilde{s}_{1}\right),\left(\mathbf{z}, \tilde{s}_{2}\right)$ and $\left(\mathbf{v}_{m}, \tilde{s}_{2}\right)$, we can respectively convert the constraints (19a) and (19b) into the following convex forms as

$$
\begin{aligned}
& \sum_{j \neq k} \mathbf{w}_{j}^{H} \mathbf{H}_{c, k} \mathbf{w}_{j}+\mathbf{z}^{H} \mathbf{H}_{c, k} \mathbf{z}+\sum_{m=1}^{M}\left(\mathbf{v}_{m}^{H} \mathbf{G}_{c, k} \mathbf{v}_{m}\right)+\sigma_{a, k}^{2}+\frac{\delta_{p, k}^{2}}{\rho_{k}} \leq F_{\mathbf{H}_{c, k}, 1}\left(\mathbf{w}_{k}, s_{1}, \tilde{\mathbf{w}}_{k}, \tilde{s}_{1}\right), \\
& \sigma_{e, l}^{2}+\sum_{j=1}^{K} \mathbf{w}_{j}^{H} \overline{\mathbf{H}}_{e, l} \mathbf{w}_{j}+\mathbf{z}^{H} \overline{\mathbf{H}}_{e, l} \mathbf{z}+\sum_{m=1}^{M}\left(\mathbf{v}_{m}^{H} \overline{\mathbf{G}}_{e, l} \mathbf{v}_{m}\right) \leq \sigma_{e, l}^{2}\left(\frac{2}{\tilde{s}_{2}}-\frac{s_{2}}{\tilde{s}_{2}^{2}}\right) \\
& +\sum_{j \neq k} F_{\overline{\mathbf{H}}_{e, l}, 0}\left(\mathbf{w}_{j}, s_{2}, \tilde{\mathbf{w}}_{j}, \tilde{s}_{2}\right)+F_{\overline{\mathbf{H}}_{e, l}, 0}\left(\mathbf{z}, s_{2}, \tilde{\mathbf{z}}, \tilde{s}_{2}\right)+\sum_{m=1}^{M} F_{\overline{\mathbf{G}}_{e, l}, 0}\left(\mathbf{v}_{m}, s_{2}, \tilde{\mathbf{v}}_{m}, \tilde{s}_{2}\right), \forall k .
\end{aligned}
$$

Denoting $g_{s_{1}, k}=F_{\mathbf{H}_{c, k}, 1}\left(\mathbf{w}_{k}, s_{1}, \tilde{\mathbf{w}}_{k}, \tilde{s}_{1}\right)-\sigma_{a, k}^{2}-\frac{\delta_{p, k}^{2}}{\rho_{k}} \quad$ and $g_{s_{2}, l}=\sigma_{e, l}^{2}\left(\frac{2}{\tilde{s}_{2}}-\frac{s_{2}}{\tilde{s}_{2}^{2}}\right)+$ $\sum_{j \neq k} F_{\overline{\mathbf{H}}_{e, l}, 0}\left(\mathbf{w}_{j}, s_{2}, \tilde{\mathbf{w}}_{j}, \tilde{s}_{2}\right)+F_{\overline{\mathbf{H}}_{e, l}, 0}\left(\mathbf{z}, s_{2}, \tilde{\mathbf{z}}, \tilde{s}_{2}\right)+\sum_{m=1}^{M} F_{\overline{\mathbf{G}}_{e, l},}\left(\mathbf{v}_{m}, s_{2}, \tilde{\mathbf{v}}_{m}, \tilde{\mathbf{s}}_{2}\right)-\sigma_{e, l^{\prime}}^{2}$ (22a) and (22b) can be rewritten as the second-order cone (SOC) constraints as

$$
\begin{aligned}
& \left\|\left[2 \mathbf{w}_{1}^{H} \mathbf{H}_{c, k}, \ldots, 2 \mathbf{w}_{k-1}^{H} \mathbf{H}_{c, k}, 2 \mathbf{w}_{k+1}^{H} \mathbf{H}_{c, k}, \ldots, 2 \mathbf{w}_{K}^{H} \mathbf{H}_{c, k}, 2 \mathbf{z}^{H} \mathbf{H}_{c, k}, 2 \mathbf{v}_{1}^{H} \mathbf{G}_{c, k}, \ldots, 2 \mathbf{v}_{M}^{H} \mathbf{G}_{c, k}, g_{s_{1}, k}-1\right]^{T}\right\| \\
& \quad \leq g_{s_{1}, k}+1, \\
& \left\|\left[2 \mathbf{w}_{1}^{H} \overline{\mathbf{H}}_{e, l}, \ldots, 2 \mathbf{w}_{K}^{H} \overline{\mathbf{H}}_{e, l}, 2 \mathbf{z}^{H} \overline{\mathbf{H}}_{e, l}, 2 \mathbf{v}_{1}^{H} \overline{\mathbf{G}}_{e, l}, \ldots, 2 \mathbf{v}_{M}^{H} \overline{\mathbf{G}}_{e, l}, g_{s_{2}, l}-1\right]^{T}\right\| \leq g_{s_{2}, l}+1 .
\end{aligned}
$$

Moreover, the constraints $(9 \mathrm{c})$ and $(9 \mathrm{~d})$ can be reformulated as

$$
\begin{gathered}
\sum_{j=1}^{K} \mathbf{w}_{j}^{H} \mathbf{H}_{c, k} \mathbf{w}_{j}+\mathbf{z}^{H} \mathbf{H}_{c, k} \mathbf{z}+\sum_{m=1}^{M} \mathbf{v}_{m}^{H} \mathbf{G}_{c, k} \mathbf{v}_{m} \geq \frac{\bar{E}^{c}}{\eta_{k}\left(1-\rho_{k}\right)}-\sigma_{a, k}^{2} . \\
\sum_{j=1}^{K} \mathbf{w}_{j}^{H} \overline{\mathbf{H}}_{e, l} \mathbf{w}_{j}+\mathbf{z}^{H} \overline{\mathbf{H}}_{e, l} \mathbf{z}+\sum_{m=1}^{M} \mathbf{v}_{m}^{H} \overline{\mathbf{G}}_{e, l} \mathbf{v}_{m} \geq \frac{\bar{E}^{e}}{\eta_{l}}-N_{E} \sigma_{e, l}^{2}, \forall l .
\end{gathered}
$$

Next, we will apply the SPCA method to obtain convex approximations of (24) and (25). By substituting $\mathbf{w}_{j} \triangleq \tilde{\mathbf{w}}_{j}+\Delta \mathbf{w}_{j}, \mathbf{z} \triangleq \tilde{\mathbf{z}}+\Delta \mathbf{z}$ and $\mathbf{v}_{m} \triangleq \tilde{\mathbf{v}}_{m}+\Delta \mathbf{v}_{m}$ into the LHS of (24) and (25), we have

$$
\begin{aligned}
& \sum_{j=1}^{K} \mathbf{w}_{j}^{H} \mathbf{H}_{c, k} \mathbf{w}_{j}+\mathbf{z}^{H} \mathbf{H}_{c, k} \mathbf{z}+\sum_{m=1}^{M} \mathbf{v}_{m}^{H} \mathbf{G}_{c, k} \mathbf{v}_{m} \\
= & \sum_{j=1}^{K}\left(\tilde{\mathbf{w}}_{j}+\Delta \mathbf{w}_{j}\right)^{H} \mathbf{H}_{c, k}\left(\tilde{\mathbf{w}}_{j}+\Delta \mathbf{w}_{j}\right)+(\tilde{\mathbf{z}}+\Delta \mathbf{z})^{H} \mathbf{H}_{c, k}(\tilde{\mathbf{z}}+\Delta \mathbf{z})+\sum_{m=1}^{M}\left(\tilde{\mathbf{v}}_{m}+\Delta \mathbf{v}_{m}\right)^{H} \mathbf{G}_{c, k}\left(\tilde{\mathbf{v}}_{m}+\Delta \mathbf{v}_{m}\right) \\
\geq & \sum_{j=1}^{K}\left(\tilde{\mathbf{w}}_{j}^{H} \mathbf{H}_{c, k} \tilde{\mathbf{w}}_{j}+2 \Re\left\{\tilde{\mathbf{w}}_{j}^{H} \mathbf{H}_{c, k} \Delta \mathbf{w}_{j}\right\}\right)+\tilde{\mathbf{z}}^{H} \mathbf{H}_{c, k} \tilde{\mathbf{z}}+2 \Re\left\{\tilde{\mathbf{z}}^{H} \mathbf{H}_{c, k} \Delta \mathbf{z}\right\} \\
& +\sum_{m=1}^{M} \tilde{\mathbf{v}}_{m}^{H} \mathbf{G}_{c, k} \tilde{\mathbf{v}}_{m}+2 \Re\left\{\tilde{\mathbf{v}}_{m}^{H} \mathbf{G}_{c, k} \Delta \mathbf{v}_{m}\right\}
\end{aligned}
$$

where the inequality is achieved by removing the quadratic terms $\sum_{j=1}^{K} \Delta \mathbf{w}_{j}^{H} \mathbf{H}_{k} \Delta \mathbf{w}_{j}, \Delta \mathbf{z}^{H} \mathbf{H}_{k} \Delta \mathbf{z}$ and $\sum_{m=1}^{M} \Delta \mathbf{v}_{m}^{H} \mathbf{G}_{c, k} \Delta \mathbf{v}_{m}$. 
Similarly, for the LHS of (25), it follows

$$
\begin{aligned}
& \sum_{j=1}^{K} \mathbf{w}_{j}^{H} \overline{\mathbf{H}}_{e, l} \mathbf{w}_{j}+\mathbf{z}^{H} \overline{\mathbf{H}}_{e, l} \mathbf{z}+\sum_{m=1}^{M} \mathbf{v}_{m}^{H} \overline{\mathbf{G}}_{e, l} \mathbf{v}_{m} \\
= & \sum_{j=1}^{K}\left(\tilde{\mathbf{w}}_{j}+\Delta \mathbf{w}_{j}\right)^{H} \overline{\mathbf{H}}_{e, l}\left(\tilde{\mathbf{w}}_{j}+\Delta \mathbf{w}_{j}\right)+(\tilde{\mathbf{z}}+\Delta \mathbf{z})^{H} \overline{\mathbf{H}}_{e, l}(\tilde{\mathbf{z}}+\Delta \mathbf{z})+\sum_{m=1}^{M}\left(\tilde{\mathbf{v}}_{\mathbf{m}}+\Delta \mathbf{v}_{m}\right)^{H} \overline{\mathbf{G}}_{e, l}\left(\tilde{\mathbf{v}}_{m}+\Delta \mathbf{v}_{m}\right) \\
\geq & \sum_{j=1}^{K}\left(\tilde{\mathbf{w}}_{j}^{H} \overline{\mathbf{H}}_{e, l} \tilde{\mathbf{w}}_{j}+2 \Re\left\{\tilde{\mathbf{w}}_{j}^{H} \overline{\mathbf{H}}_{l} \Delta \mathbf{w}_{j}\right\}\right)+\tilde{\mathbf{z}}^{H} \overline{\mathbf{H}}_{e, l} \tilde{\mathbf{z}}+2 \Re\left\{\tilde{\mathbf{z}}^{H} \overline{\mathbf{H}}_{e, l} \Delta \mathbf{z}\right\} \\
& +\sum_{m=1}^{M}\left(\tilde{\mathbf{v}}_{m}^{H} \overline{\mathbf{G}}_{e, l} \tilde{\mathbf{v}}_{m}+2 \Re\left\{\tilde{\mathbf{v}}_{m}^{H} \overline{\mathbf{G}}_{e, l} \Delta \mathbf{v}_{m}\right\}\right) .
\end{aligned}
$$

Based on (26) and (27), the linear approximations of the concave constraints (24) and (25) can be obtained as

$$
\begin{aligned}
& \sum_{j=1}^{K}\left(\tilde{\mathbf{w}}_{j}^{H} \mathbf{H}_{c, k} \tilde{\mathbf{w}}_{j}+2 \Re\left\{\tilde{\mathbf{w}}_{j}^{H} \mathbf{H}_{c, k} \Delta \mathbf{w}_{j}\right\}\right)+\tilde{\mathbf{z}}^{H} \mathbf{H}_{c, k} \tilde{\mathbf{z}}+2 \Re\left\{\tilde{\mathbf{z}}^{H} \mathbf{H}_{c, k} \Delta \mathbf{z}\right\} \\
& +\sum_{m=1}^{M}\left(\tilde{\mathbf{v}}_{m}^{H} \mathbf{G}_{c, k} \tilde{\mathbf{v}}_{m}+2 \Re\left\{\tilde{\mathbf{v}}_{m}^{H} \mathbf{G}_{c, k} \Delta \mathbf{v}_{m}\right\}\right) \geq \frac{\bar{E}^{c}}{\eta_{k}\left(1-\rho_{k}\right)}-\sigma_{a, k}^{2}, \forall k . \\
& \sum_{j=1}^{K}\left(\tilde{\mathbf{w}}_{j}^{H} \overline{\mathbf{H}}_{e, l} \tilde{\mathbf{w}}_{j}+2 \Re\left\{\tilde{\mathbf{w}}_{j}^{H} \overline{\mathbf{H}}_{l} \Delta \mathbf{w}_{j}\right\}\right)+\tilde{\mathbf{z}}^{H} \overline{\mathbf{H}}_{e, l} \tilde{\mathbf{z}}+2 \Re\left\{\tilde{\mathbf{z}}^{H} \overline{\mathbf{H}}_{e, l} \Delta \mathbf{z}\right\} \\
& \quad+\sum_{m=1}^{M}\left(\tilde{\mathbf{v}}_{m}^{H} \overline{\mathbf{G}}_{e, l} \tilde{\mathbf{v}}_{m}+2 \Re\left\{\tilde{\mathbf{v}}_{m}^{H} \overline{\mathbf{G}}_{e, l} \Delta \mathbf{v}_{m}\right\}\right) \geq \frac{\bar{E}^{e}}{\eta_{l}}-N_{E} \sigma_{e, l}^{2}, \forall l .
\end{aligned}
$$

Finally, by rearranging $(8 \mathrm{~d})$ as

$$
\begin{aligned}
& \left\|\left[\mathbf{w}_{1}^{T}, \ldots, \mathbf{w}_{K}^{T}, \mathbf{z}^{T}, \mathbf{v}_{1}{ }^{T}, \ldots, \mathbf{v}_{M}^{T}\right]\right\| \leq \sqrt{P}, \\
& \left\|\left[\mathbf{w}_{1}^{T}, \ldots, \mathbf{w}_{K}^{T}\right]\right\| \leq \sqrt{P_{1}} \\
& \left\|\left[\mathbf{v}_{1}{ }^{T}, \ldots, \mathbf{v}_{M}^{T}\right]\right\| \leq \sqrt{P_{2}} .
\end{aligned}
$$

Eventually, the original MSCM problem (8) is transformed into following convex form as

$$
\begin{aligned}
& \max _{\mathbf{w}_{k}, \mathbf{z}, \mathbf{v}_{m}, \rho_{k}, \gamma_{c}, \gamma_{e}, v_{m}, w_{m}, u_{m}, t_{l}, e_{l}, q_{l}, s_{1}, s_{2}} \gamma_{c}-\gamma_{e} \\
& \text { s.t. (12a), (12d), (13b), (13d), (14a), (14b), (14c), (14d), (18), (23), (28), (29), (30), } \\
& \quad 0<\rho_{c, l} \leq 1, \forall l, \Delta \mathbf{w}_{k}=\mathbf{w}_{k}-\tilde{\mathbf{w}}_{k}, \Delta \mathbf{z}=\mathbf{z}-\tilde{\mathbf{z}}, \Delta \mathbf{v}_{m}=\mathbf{v}_{m}-\tilde{\mathbf{v}}_{m} .
\end{aligned}
$$

Given $\Omega \triangleq\left\{\tilde{\mathbf{w}}_{k}, \tilde{\mathbf{z}}_{,} \tilde{\mathbf{v}}_{m}, \tilde{w}_{m}, \tilde{u}_{m}, \tilde{t}_{l}, \tilde{\gamma}_{e}, \tilde{s}_{1}, \tilde{s}_{2}\right\}$, problem (31) is a SOCP problem [43], which can be efficiently solved by convex optimization software such as CVX tool [46]. According to the SCA method and the SPCA technology, a convex approximation with the optimal solution can be updated iteratively, which implies that problem (8) can be optimally solved. We summarize the proposed SCA-SPCA-based algorithm in Algorithm 1. Note that due to the power constraints (30) at the femtocell sink node and the macrocell sink node, the objective function of the recast problem (31) always has an upper bound. Hence, the proposed SCA-SPCA-based algorithm can be guaranteed to iteratively converge to a global optimal solution, whose the convergence property is proved in the following theorem. 


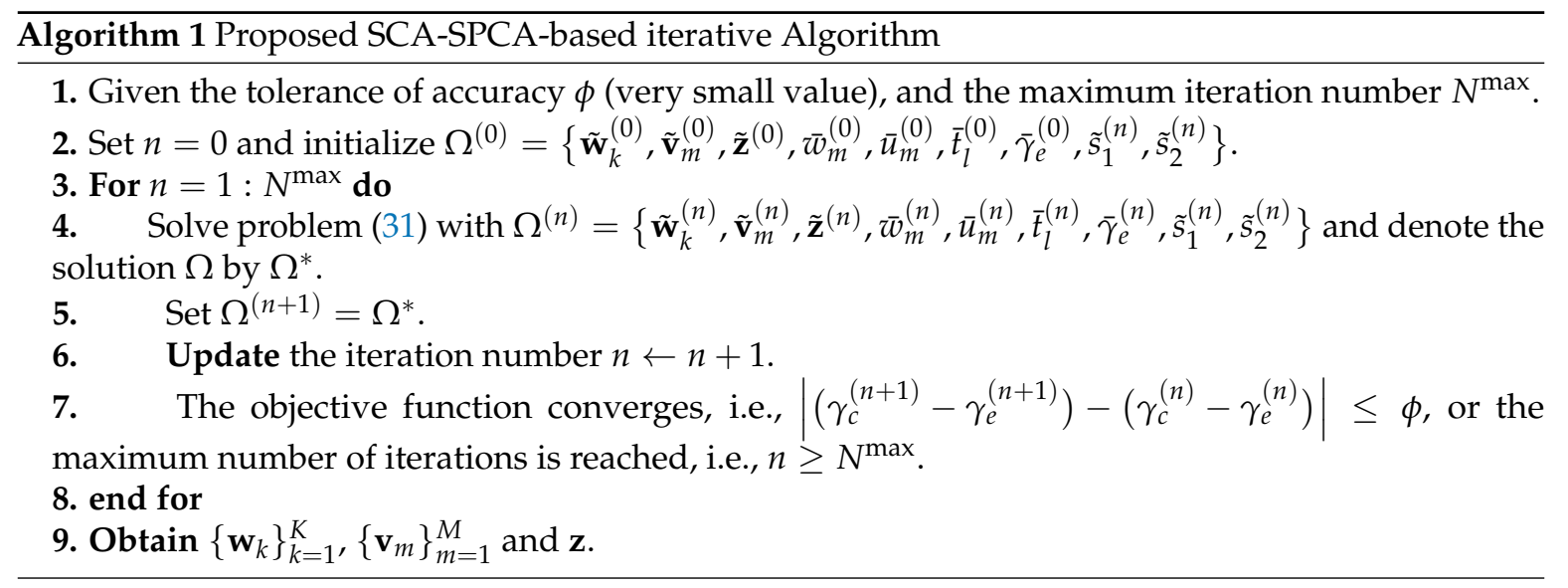

Theorem 1. Suppose that $\gamma_{c}^{(n)}$ and $\gamma_{e}^{(n)}$ are the optimal solution to problem (31) at the n-th iteration. Denoting $f_{n}^{*}\left(\gamma_{c}^{(n)}, \gamma_{e}^{(n)}\right)$ as the optimal objective function at the $q$-th iteration, we have $f_{n+1}^{*}\left(\gamma_{c}^{(n+1)}, \gamma_{e}^{(n+1)}\right) \geq$ $f_{n}^{*}\left(\gamma_{c}^{(n)}, \gamma_{e}^{(n)}\right)$. Thus, with the increase of $n,\left\|\Delta \mathbf{w}_{k}\right\|,\|\Delta \mathbf{z}\|$ and $\left\|\Delta \mathbf{v}_{m}\right\|$ approach 0. Hence, Algorithm 1 will converge.

Proof. See Appendix B.

\section{Simulation Results}

In this section, the simulation results are provided to validate the performance of our proposed SCA-SPCA-based iterative algorithm. The system simulation parameters that we have considered for the simulations are summarized in Table 2. We assume that one smallcell is in the coverage area of a macrocell, where we set the radius of the macrocell and the smallcell to $20 \mathrm{~m}$ and $10 \mathrm{~m}$, respectively [17]. Thus, the channel $\mathbf{g}_{m}, \mathbf{g}_{c, k}, \mathbf{G}_{e, l} \mathbf{h}_{m}, \mathbf{h}_{c, k}$, and $\mathbf{H}_{e, l}$ are modelled as $\mathbf{g}_{m}=H\left(g_{m}\right) \overline{\mathbf{g}}_{I}$, $\mathbf{g}_{c, k}=H\left(g_{c, k}\right) \hat{\mathbf{g}}_{I}, \mathbf{G}_{e, l}=H\left(g_{e, l}\right) \overline{\mathbf{G}}_{I}, \mathbf{h}_{m}=H\left(h_{m}\right) \overline{\mathbf{h}}_{I}, \mathbf{h}_{c, k}=H\left(h_{c, k}\right) \hat{\mathbf{h}}_{I}$, and $\mathbf{H}_{e, l}=H\left(h_{e, l}\right) \overline{\mathbf{H}}_{I}$, where $H(d)=\frac{c}{4 \pi f_{c}}\left(\frac{1}{d}\right)^{\frac{\kappa}{2}}, \overline{\mathbf{g}}_{I} \sim \mathcal{C N}(0, \mathbf{I}), \hat{\mathbf{g}}_{I} \sim \mathcal{C N}(0, \mathbf{I}), \overline{\mathbf{G}}_{I} \sim \mathcal{C N}(0, \mathbf{I}), \overline{\mathbf{h}}_{I} \sim \mathcal{C N}(0, \mathbf{I}), \hat{\mathbf{h}}_{I} \sim \mathcal{C N}(0, \mathbf{I})$, and $\overline{\mathbf{H}}_{I} \sim \mathcal{C N}(0, \mathbf{I})$, respectively. In the simulation results, we will compare the following optimization design schemes: the proposed algorithm, the MSCM scheme with fixed $\rho_{k}=0.5$, the non-AN MSCM scheme which is obtained by setting $\mathbf{z}=\mathbf{0}$, the null-AN MSCM scheme which means the AN vector lie in the null space of the HP-SNs.

Table 2. System Simulation Parameters.

\begin{tabular}{cc} 
Number of M-SN, $M$ & 3 \\
Number of HP-SN, $K$ & 2 \\
Number of LP-SN, $L$ & 2 \\
Number of LP-SN antenna, $N_{E}$ & 2 \\
Number of transmit antenna at macrocell sink node, $N_{M}$ & 4 \\
Number of transmit antenna at femtocell sink node, $N_{F}$ & 16 \\
Distance between the macrocell sink node and the M-SN, $g_{m}$ & $10 \mathrm{~m}$ \\
Distance between the macrocell sink node and the HP-SN, $g_{c, k}$ & $5 \mathrm{~m}$ \\
Distance between the macrocell sink node and the LP-SN, $g_{e, l}$ & $3 \mathrm{~m}$ \\
Distance between the femtocell sink node and the M-SN, $h_{m}$ & $6 \mathrm{~m}$ \\
Distance between the femtocell sink node and the HP-SN, $h_{c, k}$ & $5 \mathrm{~m}$ \\
Distance between the femtocell sink node and the LP-SN, $h_{e, l}$ & $2 \mathrm{~m}$ \\
Speed of light, $c$ & $3 \times 10^{8} \mathrm{~ms}{ }^{-1}$ \\
Carrier frequency, $f_{c}$ & $900 \mathrm{MHz}$ \\
Path loss exponent, $\kappa$ & 2.7 \\
Gaussian noise power at all the M-SN, $\sigma_{m}^{2}$ & $-90 \mathrm{dBm}$ \\
Gaussian noise power at all the HP-SN, $\sigma_{c, k}^{2}$ & $-50 \mathrm{dBm}$ \\
Gaussian noise power of all the LP-SNs, $\sigma_{e, l}^{2}$ & $-90 \mathrm{dBm}$ \\
Additional noise power of all the HP-SNs, $\delta_{p, k}^{2}$ & $-90 \mathrm{dBm}$ \\
Energy conversion efficiency at all the HP-SN and LP-SN, $\eta_{c, k}=\eta_{e, l}$ & 0.3 \\
\hline
\end{tabular}


In Figure 3, we illustrate the convergence performance of the proposed SCA-SPCA-based iterative algorithm with respect to (w.r.t.) iteration numbers. We set $\bar{R}^{c}=1 \mathrm{bps} / \mathrm{Hz}, \bar{E}^{c}=10 \mathrm{dBm}, \bar{E}^{e}=10 \mathrm{dBm}$, $P=60 \mathrm{dBm}$ and $P_{1}=40 \mathrm{dBm}$. It is easily seen from Figure 3 that the convergence of all cases can be quickly achieved within three iterations. It is observed that the average minimal secrecy capacity (MSC) at the M-SN becomes great as $P_{2}$ increases.

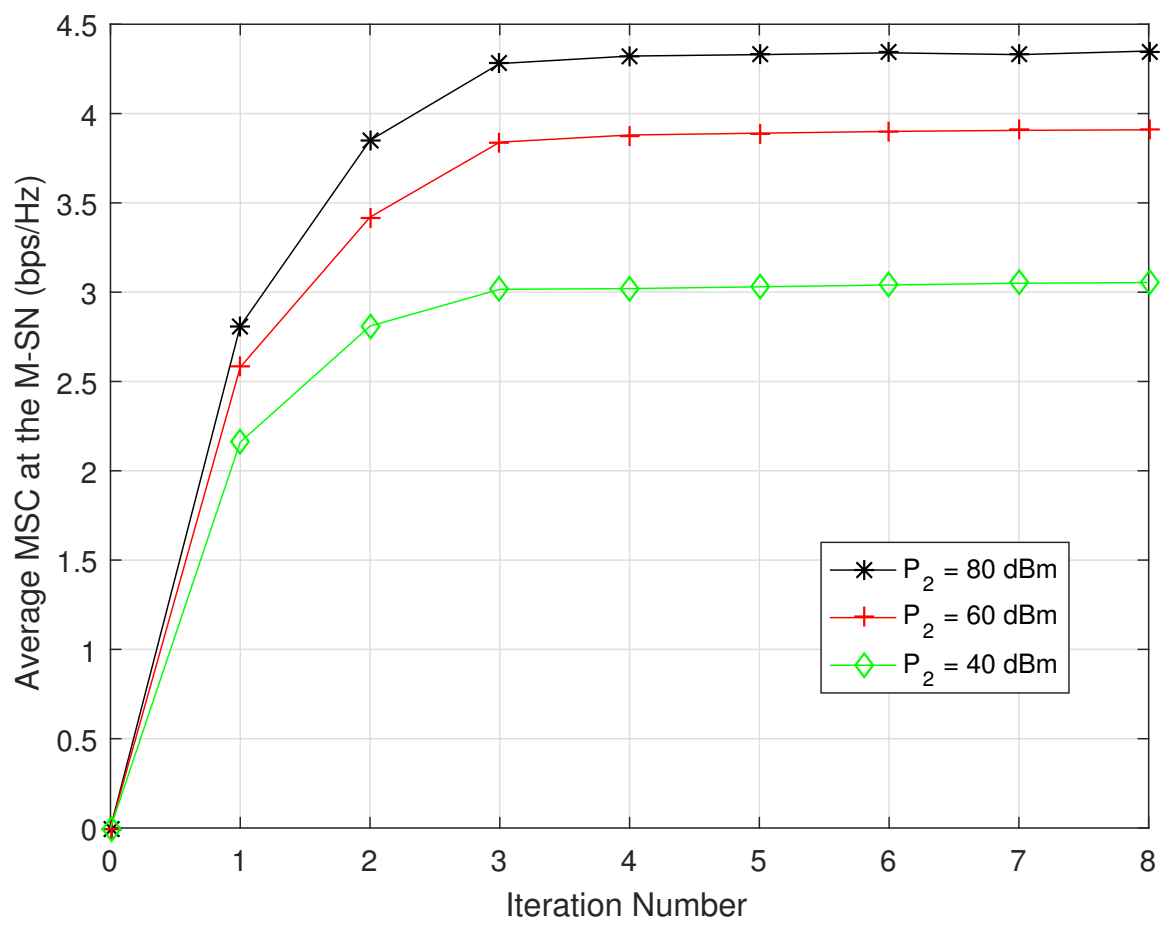

Figure 3. Average MSC at the M-SN with respect to iteration number.

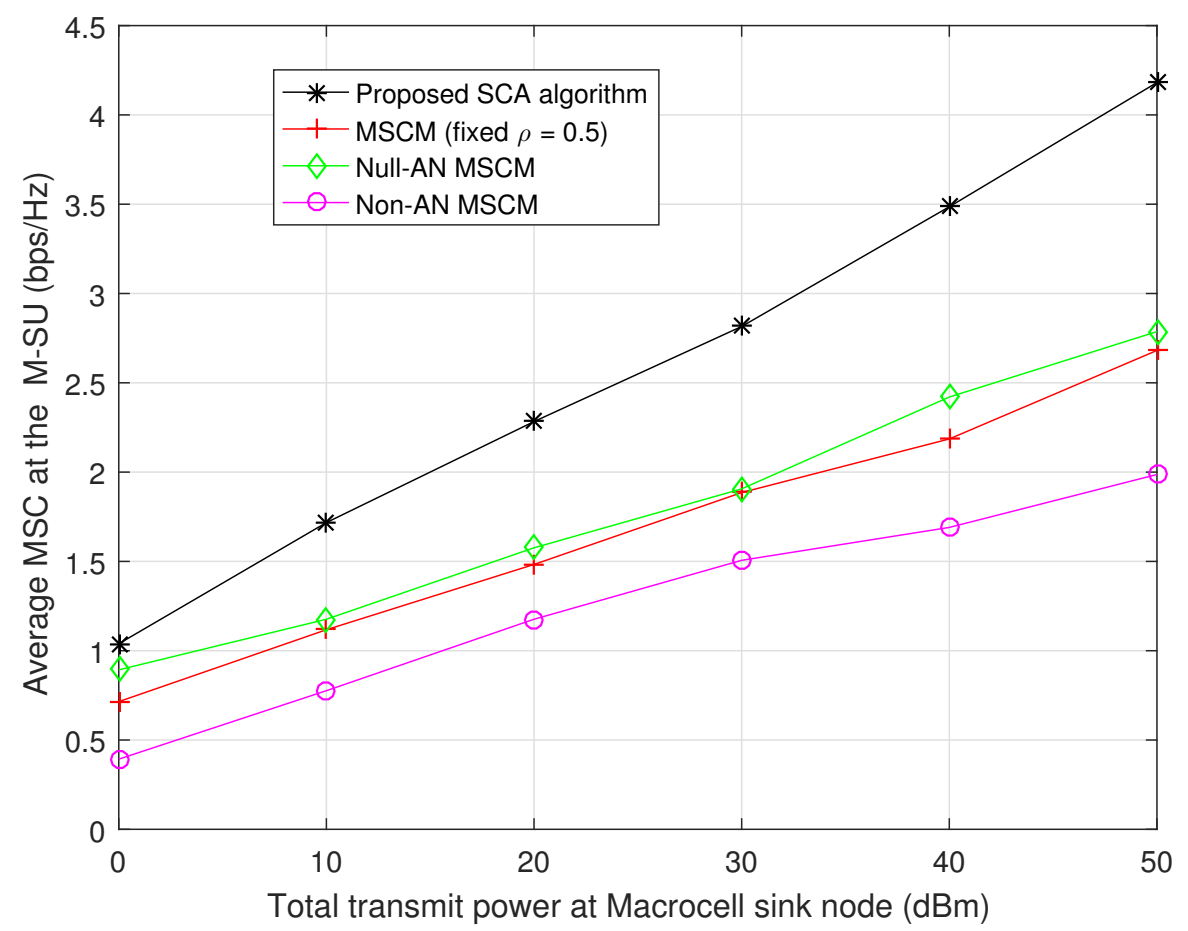

Figure 4. Average MSC at the M-SN with respect to the total transmit power at macrocell sink node. 


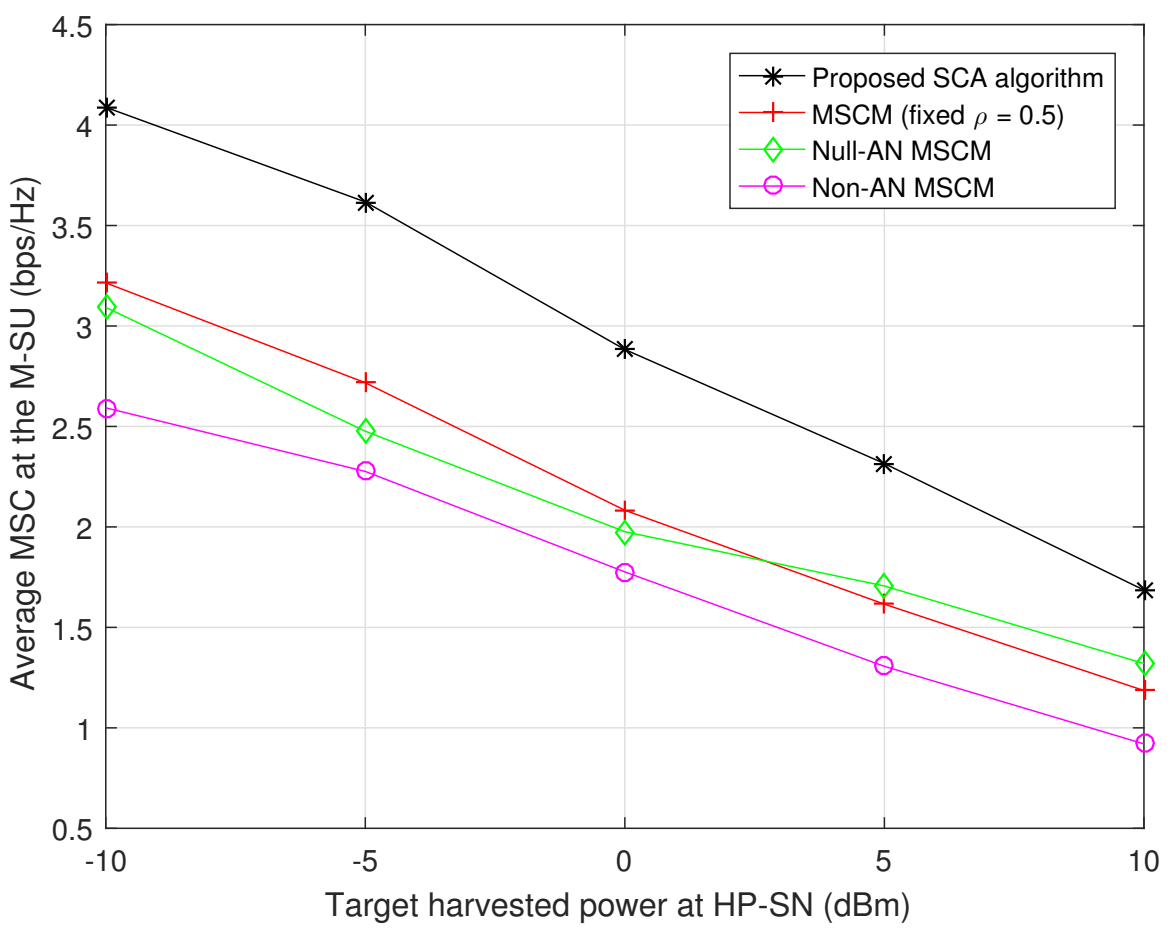

Figure 5. Average MSC at the M-SN with respect to the target harvested power at HP-SN.

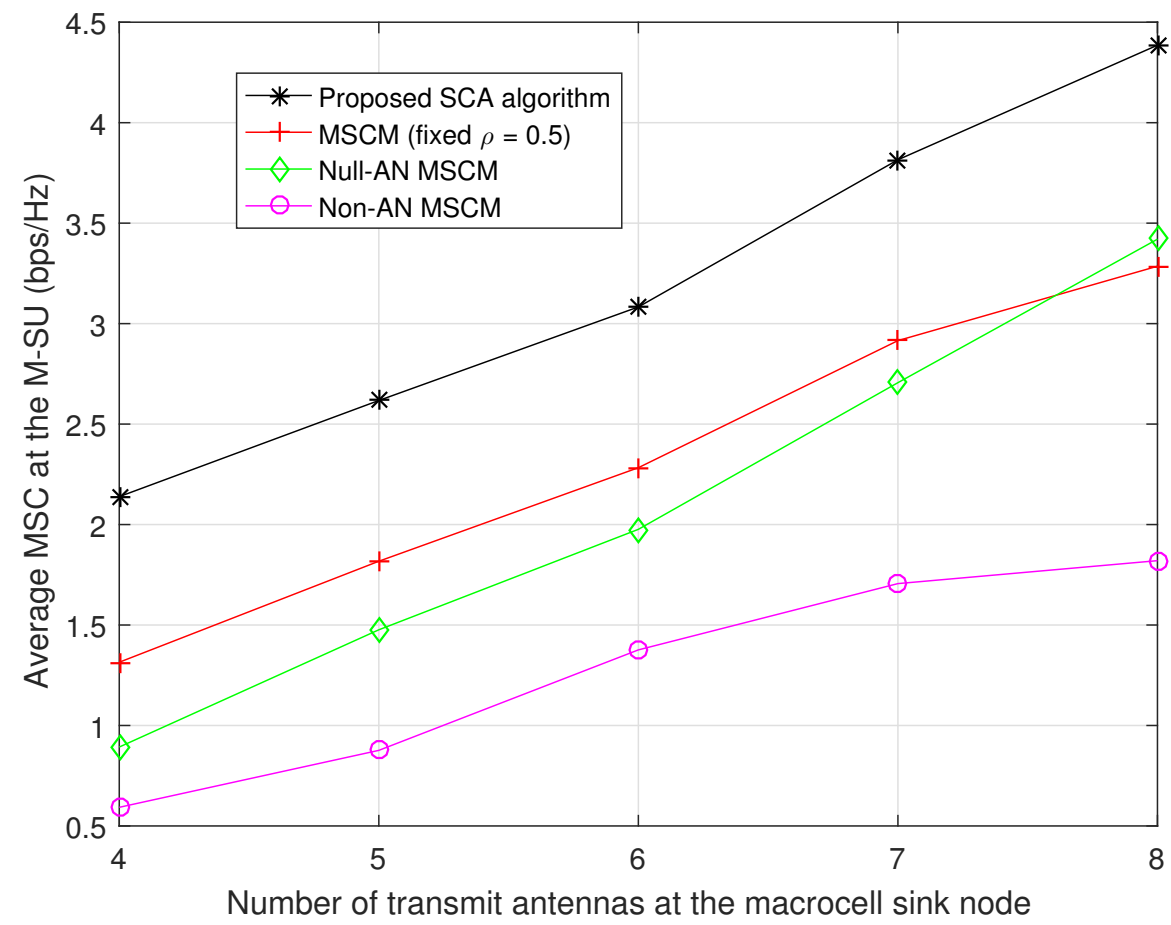

Figure 6. Average MSC at the M-SN with respect to the number of transmit antennas at the macrocell sink node. 


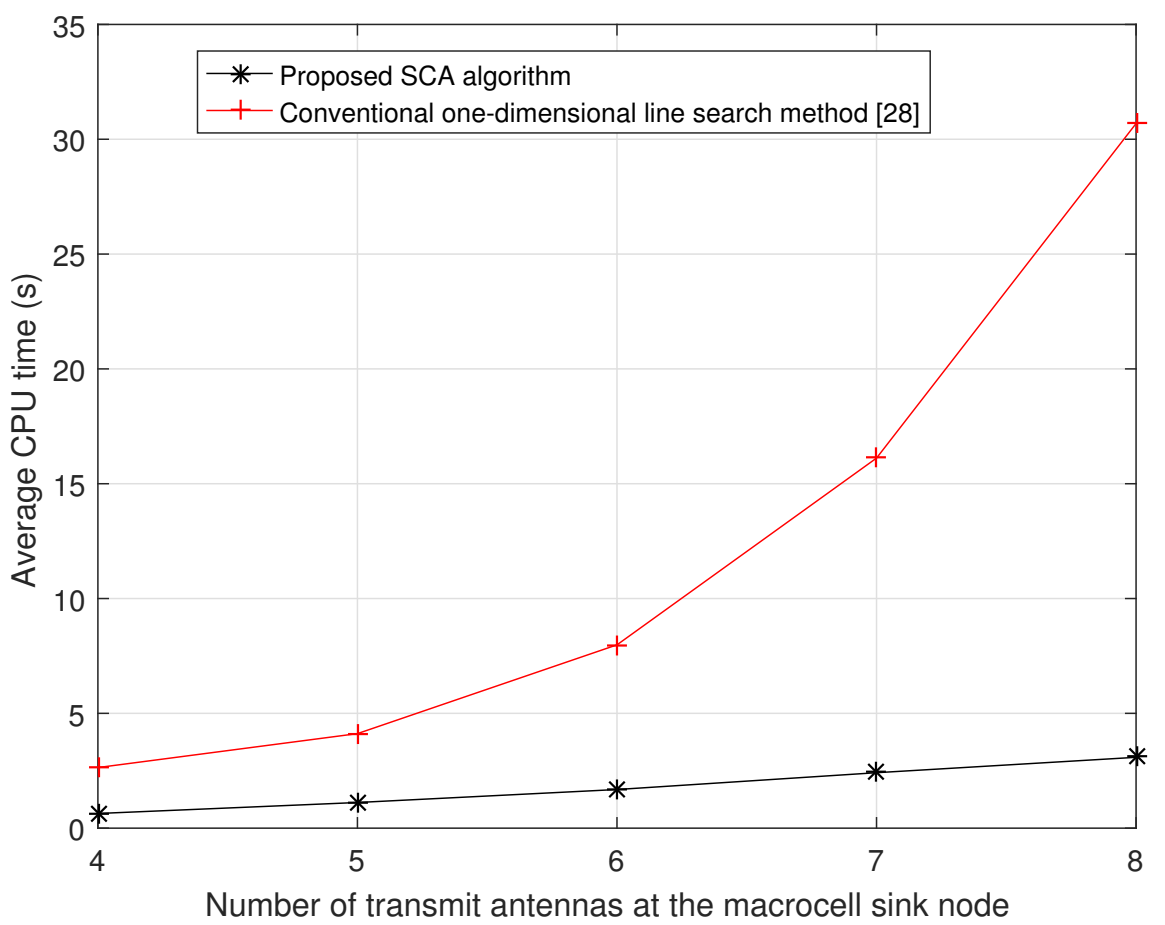

Figure 7. Average CPU time with respect to the number of transmit antennas at the macrocell sink node.

Figure 4 illustrates the average MSC at the M-SN in terms of different target transmit power at macrocell sink node with $\bar{R}^{c}=1.5 \mathrm{bps} / \mathrm{Hz}, \bar{E}^{c}=5 \mathrm{dBm}, \bar{E}^{e}=5 \mathrm{dBm}, P=60 \mathrm{dBm}$ and $P_{1}=40 \mathrm{dBm}$ over 500 feasible channel realizations. It is observed that the average MSC increases with the target transmit power at macrocell sink node. In addition, the proposed SCA-SPCA-based algorithm outperforms all the conventional baseline schemes. Moreover, we can check that the proposed SCA-SPCA-based algorithm performs better than the non-AN MSCM, null- AN MSCM and the MSCM scheme with $\rho_{k}=0.5$, and the performance gap is always gradually increasing regardless of the target transmit power at macrocell sink node. This reveals that optimizing the PS ratio $\rho_{k}$ is important.

In Figure 5, we compare the average MSC at the M-SN w.r.t. the different target-harvested power at HP-SN by fixing $\bar{R}^{c}=1 \mathrm{bps} / \mathrm{Hz}, \bar{E}^{e}=10 \mathrm{dBm}, P=70 \mathrm{dBm}, P_{1}=50 \mathrm{dBm}$ and $P_{2}=60 \mathrm{dBm}$. In this figure, we can observe that all schemes decrease with the increase of $\bar{E}^{c}$. Moreover, the proposed SCA-SPCA-based algorithm always perform better in average MSC than all three conventional baseline schemes. Compared with the non-AN MSCM and the MSCM scheme with $\rho_{k}=0.5$, the averageMSC of the proposed AN-aidedalgorithm is $1.2 \mathrm{bps} / \mathrm{Hz}$ and $0.8 \mathrm{bps} / \mathrm{Hz}$ higher at $0 \mathrm{dBm}$. It is observed that the proposed algorithm outperforms the null-AN MSCM.

In Figure 6, we plot the average MSC at the M-SN in terms of different number of transmit antennas at the macrocell sink node with $\bar{R}^{c}=0.5 \mathrm{bps} / \mathrm{Hz}, \bar{E}^{c}=5 \mathrm{dBm}, \bar{E}^{e}=10 \mathrm{dBm}, P=60 \mathrm{dBm}$, $P_{1}=40 \mathrm{dBm}$ and $P_{2}=60 \mathrm{dBm}$. We can check that the curves of the proposed SCA-SPCA-based algorithm and the MSCM scheme with fixed $\rho_{k}=0.5$ increase with the same slope. Moveover, when $N_{M}$ increases, the performance gap between the non-AN MSCM scheme and the proposed algorithm becomes wider. This indicates that the AN technology is essential in obtaining the performance gains. Furthermore, the proposed algorithm outperforms the null-AN MSCM scheme by $0.8 \mathrm{bps} / \mathrm{Hz}$.

In Figure 7 , in terms of the average central processing unit (CPU) running time, the computational complexities of the proposed method and the conventional one-dimensional line search (1-D) method are compared for a channel realization under various MF on a computer (The CPU is Intel Core i7-8500U $1.8 \mathrm{GHz}$, and the size of random access memory (RAM) is $8 \mathrm{~GB}$ ). In this simulation, we set $\bar{R}^{c}=1 \mathrm{bps} / \mathrm{Hz}, \bar{E}^{c}=50 \mathrm{dBm}, \bar{E}^{e}=0 \mathrm{dBm}, P=80 \mathrm{dBm}, P_{1}=40 \mathrm{dBm}$ and $P_{2}=60 \mathrm{dBm}$. We can see that our proposed algorithm has much lower complexity than the conventional 1-D method in [29]. 


\section{Conclusions}

Considering the security transmission with PS-enabled SWIPT approach, we have investigated the AN-based beamforming design problem in a two-tier MIMO HWSN where a femtocell is underlaying a macrocell. The jointly design problem of the transmit beamforming vectors, the AN and PS ratio has been modeled to maximize the MSC of the M-SNs subject to the secrecy capacity constraint, the harvested energy constraint and the transmit power constraint. To reduce the performance loss from the rank relaxation, an novel SCA-SPCA-based iterative algorithm has been proposed and its convergence property is proved. Numerical results have demonstrated that the proposed SCA-SPCA-based algorithm always outperforms the conventional non-AN, null-AN and "fixed PS ratio" schemes. As a future work, the proposed algorithm can be extended to a multi femtocell scenario in a macrocell. In that case, interference between femtocells needs to be considered. In addition, the dependency of non-linear power-splitting EH model and EH efficiency on the received user characteristic can be an extension of this work.

Author Contributions: Conceptualization, Z.Z. and W.H.; Methodology, W.G., Z.Z. and Z.C.; Software, W.G., Z.Z. and Y.W.; Formal analysis, W.G., Z.Z. and Z.C.; Supervision, Z.W.; Writing-original draft preparation, Z.Z. and W.G.; Writing-review and editing, Z.Z. and W.G.; Visualization Q.W. and W.G.

Funding: This work was supported in part by the National Nature Science Foundation of China under grants 61801434, 61571402, 61771431 and 61801435, in part by the Project funded by China Postdoctoral Science Foundation under Grant 2018M642784, in part by the Scientific and Technological Key Project of Henan Province under Grant 192102310178, 192102210246, in part by the National Science and Technology Major Project under grant 2017ZX03001001. This work of Q. Wu was supported in part by the National Natural Science Foundation of China under Grant No. 61701197, the Project funded by China Postdoctoral Science Foundation under Grant No. 2018M641354, 2018M633733, the Open Research Fund of National Mobile Communications Research Laboratory, Southeast University, under Grant No. 2018D15. This work of Z. Chu was supported in part by the Open Fund of the Shaanxi Key Laboratory of Information Communication Network and Security under Grant ICNS201801.

Conflicts of Interest: The authors declare no conflict of interest.

$\begin{array}{ll}\text { Abbreviations } \\ \text { The following abbreviations are used in this manuscript: } \\ \text { HWSN } & \text { heterogeneous wireless sensor networks } \\ \text { SWIPT } & \text { simultaneous wireless information and power transfer } \\ \text { AN } & \text { artificial noise } \\ \text { SCA } & \text { successive convex approximation } \\ \text { SPCA } & \text { sequential parametric convex approximation } \\ \text { SOCP } & \text { second order cone programming } \\ \text { IoT } & \text { Internet-of-Things } \\ \text { WSN } & \text { wireless sensor network } \\ \text { MBS } & \text { macrocell base station } \\ \text { FBS } & \text { femtocell base station } \\ \text { SN } & \text { sensor node } \\ \text { HP-SN } & \text { high-priority sensor node } \\ \text { LP-SN } & \text { low-priority sensor node } \\ \text { M-SN } & \text { macrocell sensor node } \\ \text { HetNet } & \text { heterogeneous networks } \\ \text { WPT } & \text { wireless power transmission } \\ \text { WIT } & \text { wireless information transmission } \\ \text { MISO } & \text { multiple-input single-output } \\ \text { MIMO } & \text { multiple-input multiple } \\ \text { CoMP } & \text { coordinated multi-point } \\ \text { CSI } & \text { channel state imformation } \\ \text { PLS } & \text { physical-layer security } \\ \text { PS } & \text { power splitting } \\ \text { MSC } & \text { minimal secrecy capacity }\end{array}$


MSCM MSC maximization

AWGN additive white Gaussian noise)

ID imformation decoding

EH energy harvesting

QoL quadratic-over-linear

\section{Appendix A}

Proof of Lemma 1. Let $a=\operatorname{rank}(\mathbf{A})$. The case of $a \geq 0$ is trivial. For $a \geq 1$, let $\lambda_{1} \geq \lambda_{2} \geq \ldots \geq \lambda_{a}>0$ denote the nonzero eigenvalues of $\mathbf{A}$. It follows

$$
\begin{aligned}
|\mathbf{I}+\mathbf{A}| & =\prod_{i=1}^{a}\left(1+\lambda_{i}\right) \\
& =1+\prod_{i=1}^{a} \lambda_{i}+\prod_{i \neq k}^{a} \lambda_{i} \lambda_{k}+\ldots \\
& \geq 1+\prod_{i=1}^{a} \lambda_{i}=1+\operatorname{tr}(\mathbf{A})
\end{aligned}
$$

and it can be seen that the inequality above holds if and only if $a=1$.

\section{Appendix B}

Proof of Theorem 1. Since problem (31) is convex, the optimal solution $\Omega^{(n)}$ is obtained by solving (31) for a given $\Omega^{(n-1)}$. Due to the solution $\Omega^{(n-1)}$ obtained in the $n$-th iteration, $\Omega^{(n)}$ can be updated in the $(n+1)$-th iteration. Based on (28) and (29), we can obtain the following inequalities in the $(n+1)$-th iteration.

$$
\begin{aligned}
& \sum_{j=1}^{K}\left(\mathbf{w}_{j}{ }^{H} \mathbf{H}_{c, k} \mathbf{w}_{j}\right)+\mathbf{z}^{H} \mathbf{H}_{c, k} \mathbf{z}+\sum_{m=1}^{M}\left(\mathbf{v}_{m}^{H} \mathbf{G}_{c, k} \mathbf{v}_{m}\right) \\
\geq & \sum_{j=1}^{K}\left(\tilde{\mathbf{w}}_{j}^{* H} \mathbf{H}_{c, k} \tilde{\mathbf{w}}_{j}+2 \Re\left\{\tilde{\mathbf{w}}_{j}^{* H} \mathbf{H}_{c, k} \Delta \mathbf{w}_{j}\right\}\right)+\tilde{\mathbf{z}}^{* H} \mathbf{H}_{c, k} \tilde{\mathbf{z}}+2 \Re\left\{\tilde{\mathbf{z}}^{* H} \mathbf{H}_{c, k} \Delta \mathbf{z}\right\} \\
& +\sum_{m=1}^{M}\left(\tilde{\mathbf{v}}_{m}^{* H} \mathbf{G}_{c, k} \tilde{\mathbf{v}}_{m}+2 \Re\left\{\tilde{\mathbf{v}}_{m}^{* H} \mathbf{G}_{c, k} \Delta \mathbf{v}_{m}\right\}\right) \\
\geq & \frac{\bar{E}^{c}}{\eta_{k}\left(1-\rho_{k}\right)}-\sigma_{a, k}^{2}, \forall k, \\
& \sum_{j=1}^{K}\left(\mathbf{w}_{j}{ }^{H} \overline{\mathbf{H}}_{e, l} \mathbf{w}_{j}\right)+\mathbf{z}^{H H} \overline{\mathbf{H}}_{e, l} \mathbf{z}+\sum_{m=1}^{M}\left(\mathbf{v}_{m}{ }^{H} \overline{\mathbf{G}}_{e, l} \mathbf{v}_{m}\right) \\
\geq & \sum_{j=1}^{K}\left(\tilde{\mathbf{w}}_{j}^{* H} \overline{\mathbf{H}}_{e, l} \tilde{\mathbf{w}}_{j}+2 \Re\left\{\tilde{\mathbf{w}}_{j}^{* H} \overline{\mathbf{H}}_{l} \Delta \mathbf{w}_{j}\right\}\right)+\tilde{\mathbf{z}}^{* H} \overline{\mathbf{H}}_{e, l} \tilde{\mathbf{z}}+2 \Re\left\{\tilde{\mathbf{z}}^{* H} \overline{\mathbf{H}}_{e, l} \Delta \mathbf{z}\right\} \\
& +\sum_{m=1}^{M}\left(\tilde{\mathbf{v}}_{m}^{* H} \overline{\mathbf{G}}_{e, l} \tilde{\mathbf{v}}_{m}+2 \Re\left\{\tilde{\mathbf{v}}_{m}^{* H} \overline{\mathbf{G}}_{e, l} \Delta \mathbf{v}_{m}\right\}\right) \\
\geq & \frac{\bar{E}^{e}}{\eta_{l}}-N_{E} \sigma_{e, l}^{2}, \forall l,
\end{aligned}
$$

which are conservative convex approximations with $\mathbf{w}_{j}=\tilde{\mathbf{w}}_{j}^{*}+\Delta \mathbf{w}_{j}, \mathbf{z}=\tilde{\mathbf{z}}^{*}+\Delta \mathbf{z}$ and $\mathbf{v}_{m}=\tilde{\mathbf{v}}_{m}^{*}+\Delta \mathbf{v}_{m}$.

According to the linear approximations employed in (A1) and (A2), the rule for updating in Algorithm 1 ensures that $\left\{\boldsymbol{w}_{j}^{*}\right\},\left\{\boldsymbol{z}^{*}\right\}$ and $\left\{\boldsymbol{v}_{m}^{*}\right\}$ has always been feasible to problem (31) at the $(q+1)$-th iteration. Thus, we immediately have $f_{n+1}^{*}\left(\gamma_{c}^{(n+1)}, \gamma_{e}^{(n+1)}\right) \geq f_{n}^{*}\left(\gamma_{c}^{(n)}, \gamma_{e}^{(n)}\right)$. In other words, this behaviour of $\Omega^{(n)}$ reveals that the proposed algorithm has always a monotonically non-decreasing 
sequence of the objective values. On account of the transmit power constraint at the macrocell sink node and femtocell sink node, problem (31) has an upper bound on the MSC. Therefore, the proposed algorithm can be guaranteed to converge to some optimum solution, i.e., $\left\|\Delta \mathbf{w}_{j}\right\|=0,\|\Delta \mathbf{z}\|=0$, $\left\|\Delta \mathbf{v}_{m}\right\|=0$, and $f_{n+1}^{*}\left(\gamma_{c}^{(n+1)}, \gamma_{e}^{(n+1)}\right)=f_{n}^{*}\left(\gamma_{c}^{(n)}, \gamma_{e}^{(n)}\right)$.

\section{References}

1. Wang, S.; Wan, J.; Zhang, D.; Li, D.; Zhang, C. Towards smart factory for industry 4.0: A self-organized multi-agent system with big data based feedback and coordination. Comput. Netw. 2016, 101, 158-168. [CrossRef]

2. Williams, J.M.; Khanna, R.; Ruiz-Rosero, J.P.; Pisharody, G.; Qian, Y.; Carlson, C.R.; Liu, H.; Ramirez-Gonzalez, G. Weaving the wireless Web: Toward a low-power, dense wireless sensor network for the industrial IoT. IEEE Microw. Mag. 2017, 18, 40-63. [CrossRef]

3. Khan, I.; Belqasmi, F.; Glitho, R.; Crespi, N.; Morrow, M.; Polakos, P. Wireless sensor network virtualization: A survey. IEEE Commun. Surv. Tutor. 2015, 18, 553-576. [CrossRef]

4. Liu, H.-H.; Su, J.-J.; Chou, C.-F. On energy-effcient straight-line routing protocol for wireless sensor networks. IEEE Syst. J. 2017, 11, 2374-2382. [CrossRef]

5. Chincoli, M.; Liotta, A. Self-learning power control in wireless sensor networks. Sensors 2018, 18, 375. [CrossRef]

6. Wang, C.X.; Haider, F.; Gao, X.; You, X.-H.; Yang, Y.; Yuan, D.; Aggoune, H.M.; Haas, H.; Fletcher, S.; Hepsaydir, E. Cellular architecture and key technologies for 5G wireless communication networks. IEEE Commun. Mag. 2014, 52, 122-130. [CrossRef]

7. Xiong, K.; Zhang, Y.; Fan, P.; Yang, H. Evaluation framework for user experience in 5G systems: On systematic rateless-coded transmissions. IEEE Access 2016, 4, 9108-9118. [CrossRef]

8. Damnjanovic, A.; Montojo, J.; Wei, Y.; Ji, T.; Luo, T.; Vajapeyam, M.; Yoo, T.; Song, O.; Malladi, D. A survey on 3GPP heterogeneous networks. IEEE Wirel. Commun. 2011, 18, 10-21. [CrossRef]

9. Dhillon, H.S.; Ganti, R.K.; Baccelli, F.; Andrews, J.G. Modeling and analysis of K-tier downlink heterogeneous cellular networks. IEEE J. Sel. Areas Commun. 2012, 30, 550-560. [CrossRef]

10. Lopez-Perez, D.; Guvenc, I.; de la Roche, G.; Kountouris, M.; Quek, T.Q.S.; Zhang, J. Enhanced intercell interference coordination challenges in heterogeneous networks. IEEE Wirel. Commun. 2011, 18, 22-30. [CrossRef]

11. Bi, S.; Ho, C.K.; Zhang, R. Wireless powered communication: Opportunities and challenges. IEEE Commun. Mag. 2015, 53, 117-125. [CrossRef]

12. Xiong, K.; Fan, P.; Zhang, C.; Letaief, K.B. Wireless information and energy transfer for two-hop non-regenerative MIMO-OFDM relay networks. IEEE J. Sel. Areas Commun. 2015, 33, 1595-1611. [CrossRef]

13. Xiong, K.; Chen, C.; Qu, G.; Fan, P.; Letaief, K.B. Group cooperation with optimal resource allocation in wireless powered communication networks. IEEE Trans. Wirel. Commun. 2017, 16, 3840-3853. [CrossRef]

14. Zhang, R.; Ho, C. MIMO broadcasting for simultaneous wireless information and power transfer. IEEE Trans. Wirel. Commun. 2013, 12, 1989-2001. [CrossRef]

15. Xiong, K.; Wang, B.; Liu, K.J.R. Rate-energy region of SWIPT for MIMO broadcasting under nonlinear energy harvesting model. IEEE Trans. Wirel. Commun. 2017, 16, 5147-5161. [CrossRef]

16. Erol-Kantarci, M.; Mouftah, H.T. Radio-frequency-based wireless energy transfer in LTE-A heterogenous networks. In Proceedings of the IEEE Symposium on Computers and Communications (ISCC), Funchal, Portugal, 23-26 June 2014; pp. 1-6.

17. Tang, J.; Shojaeifard, A.; So, D.K.C.; Wong, K.; Zhao, N. Energy efficiency optimization for CoMP-SWIPT heterogeneous networks. IEEE Trans. Commun. 2018, 66, 6368-6383. [CrossRef]

18. Jiang, R.; Xiong, K.; Fan, P.; Zhang, Y.; Zhong, Z. Optimal design of SWIPT systems with multiple heterogeneous users under non-linear energy harvesting model. IEEE Access 2017, 5, 11479-11489. [CrossRef]

19. Chen, X.; Ng, D.W.K.; Chen, H. Secrecy wireless information and power transfer: Challenges and opportunities. IEEE Wirel. Commun. 2016, 23, 54-61. [CrossRef]

20. Khisti, A.; Wornell, G.W. Secure transmission with multiple antennas I: The MISOME wiretap channel. IEEE Trans. Inf. Theory 2010, 56, 3088-3104. [CrossRef] 
21. Shiu, Y.; Chang, S.Y.; Wu, H.; Huang, S.C.; Chen, H. Physical layer security in wireless networks: A tutorial. IEEE Wirel. Commun. 2011, 18, 66-74. [CrossRef]

22. Liu, L.; Zhang, R.; Chua, K.C. Secrecy wireless information and power transfer with MISO beamforming. IEEE Trans. Signal Process. 2014, 62, 1850-1863. [CrossRef]

23. Zhu, Z.; Chu, Z.; Wang, Z.; Lee, I. Outage constrained robust beamforming for secure broadcasting systems with energy harvesting. IEEE Trans. Wirel. Commun. 2016, 15, 7610-7620. [CrossRef]

24. Zhu, Z.; Chu, Z.; Wang, N.; Huang, S.; Wang, Z.; Lee, I. Beamforming and power splitting designs for AN-aided secure multi-user MIMO SWIPT systems. IEEE Trans. Inf. Forensics Secur. 2017, 12, 2861-2874. [CrossRef]

25. Lu, Y.; Xiong, K.; Fan, P.; Ding, Z.; Zhong, Z.; Letaief, K.B. Global energy efficiency in secure MISO SWIPT systems with non-linear power-splitting EH model. IEEE J. Sel. Areas Commun. 2019, 37, 216-232. [CrossRef]

26. Ng, D.W.K.; Lo, E.S.; Schober, R. Robust beamforming for secure communication in systems with wireless information and power transfer. IEEE Trans. Wirel. Commun. 2014, 13, 4599-4615. [CrossRef]

27. Lu, Y.; Xiong, K.; Fan, P.; Zhong, Z.; Letaief, K.B. Robust transmit beamforming with artificial redundant signals for secure SWIPT system under non-linear EH model. IEEE Trans. Wirel. Commun. 2018, 17, 2218-2232. [CrossRef]

28. Li, B.; Fei, Z.; Chu, Z. Optimal transmit beamforming for secure SWIPT in a Two-Tier HetNet. IEEE Commun. Lett. 2017, 21, 2476-2479. [CrossRef]

29. Ren, Y.; Lv, T.; Gao, H.; Li, Y. Secure wireless information and power transfer in heterogeneous networks. IEEE Access 2017, 5, 4967-4979. [CrossRef]

30. Guo, S.; Wang, F.; Yang, Y.; Xiao, B. Energy-efficient cooperative Tfor simultaneous wireless information and power transfer in clustered wireless sensor networks. IEEE Trans. Commun. 2015, 63, 4405-4417. [CrossRef]

31. Guo, S.; Shi, Y.; Yang, Y.; Xiao, B. Energy efficiency maximization in mobile wireless energy harvesting sensor networks. IEEE Trans. Mob. Comput. 2018, 17, 1524-1537. [CrossRef]

32. Pan, G.; Lei, H.; Yuan, Y.; Ding, Z. Performance analysis and optimization for SWIPT wireless sensor networks. IEEE Trans. Commun. 2017, 65, 2291-2302. [CrossRef]

33. Liu, G.; Sun, Z.; Jiang, T. Joint time and energy allocation for QoS-aware throughput maximization in MIMO-based wireless powered underground sensor networks. IEEE Trans. Commun. 2019, 67, 1400-1412. [CrossRef]

34. Ha, D.B.; So-In, C.; Tran, H.; Nguyen, T.G.; Baig, Z.A.; Sanguanpong, S. Performance analysis of DF/AF cooperative MISO wireless sensor networks with NOMA and SWIPT over nakagami- $m$ fading. IEEE Access 2018, 6, 56142-56161.

35. Hu, X.; Huang, K.; Chen, Y.; Xu, X.; Liang, X. Secrecy analysis of UL transmission for SWIPT in WSNs with densely clustered eavesdroppers. In Proceedings of the 9th International Conference on Wireless Communications and Signal Processing (WCSP), Nanjing, China, 11-13 October 2017.

36. Chae, S.H.; Jeong, C.; Lim, S.H. Simultaneous wireless information and power transfer for Internet-of-Things sensor networks. IEEE Internet Things J. 2018, 5, 2829-2843. [CrossRef]

37. Yi, B.; Xiang, M.; Yao, W.; Qiu, K.; Huang, T.; Xie, Q. Simultaneous wireless information and power transfer in wireless sensor networks with fountain codes. In Proceedings of the IEEE International Conference on Internet-of-Things (iThings), Devon, UK, 21-23 June 2017; pp. 516-521.

38. Luo, Z.-Q.; Ma, W.-K.; So, A.-C.; Ye, Y.; Zhang, S. Semidefinite relaxation of quadratic optimization problems. IEEE Trans. Signal Process. Mag. 2010, 27, 20-34.

39. Lobo, M.S.; Vandenberghe, L.; Boyd, S.; Lebret, H. Applications of Second Order Cone Programming. Linear Algebra Appl. 1998, 284, 193-228. [CrossRef]

40. Ding, Z.; Zhong, C.; Ng, D.W.; Peng, M.; Suraweera, H.A.; Schober, R.; Poor, H.V. Application of smart antenna technologies in simultaneous wireless information and power transfer. IEEE Commun. Mag. 2015, 53, 86-93. [CrossRef]

41. Beck, A.; Ben-Tal, A.; Tetruashvili, L. A sequential parametric convex approximation method with applications to nonconvex truss topology design problems. J. Glob. Optim. 2010, 47, 29-51. [CrossRef]

42. Li, Q.; Ma, W. Optimal and robust transmit designs for MISO channel secrecy by semidefinite programming. IEEE Trans. Signal Process. 2011, 59, 3799-3812. [CrossRef]

43. Boyd, S.; Vandenberghe, L. Convex Optimization; Cambridge University Press: Cambridge, UK, 2004. 
44. Chu, Z.; Cumanan, K.; Ding, Z.; Johnston, M.; le Goff, S. Secrecy rate optimizations for a MIMO secrecy channel with a cooperative jammer. IEEE Trans. Veh. Technol. 2015, 64, 1833-1847. [CrossRef]

45. Vishwanathan, A.S.; Smola, A.J.; Vishwanathan, S.V.N. Kernel methods for missing variables. In Proceedings of the 10th International Workshop on Artificial Intelligence and Statistics, Bridgetown, Barbados, 6-8 January 2005; pp. 325-332.

46. Grant, M.; Boyd, S. CVX: Matlab Software for Disciplined Convex Programming. 2012. Available online: http:/ / cvxr.com/cvx (accessed on 8 August 2018).

(C) 2019 by the authors. Licensee MDPI, Basel, Switzerland. This article is an open access article distributed under the terms and conditions of the Creative Commons Attribution (CC BY) license (http:/ / creativecommons.org/licenses/by/4.0/). 